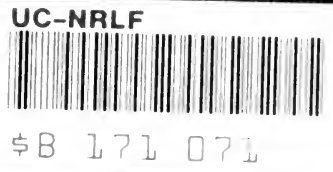




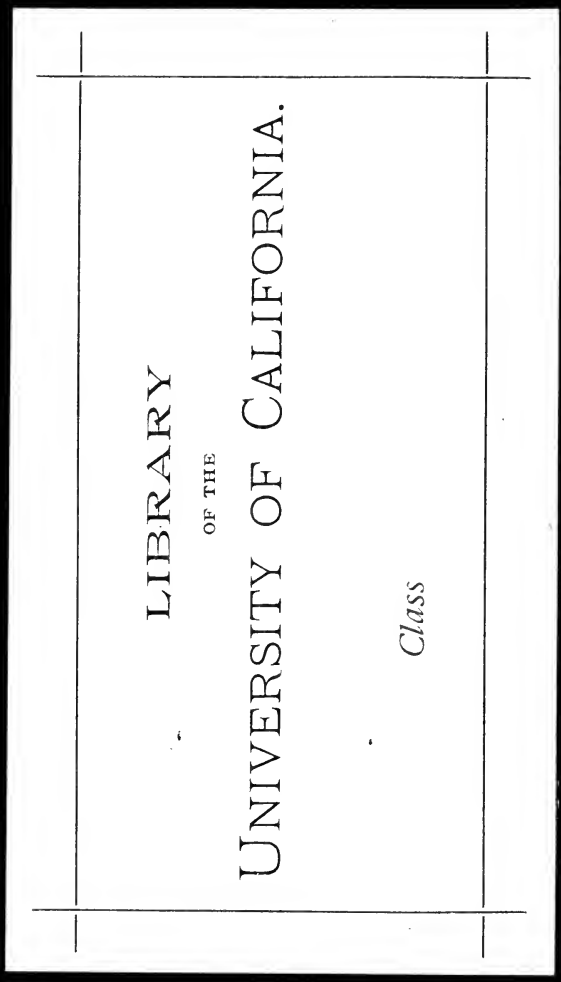




\section{-}




Digitized by the Internet Archive in 2007 with funding from Microsoft Corporation 


\title{
STATISTICAL ABSTRACT
}

\section{OF THE WORLD}

\author{
BY \\ HENRY GANNETT
}

FIRST EDITION

FIRST THOUSAND

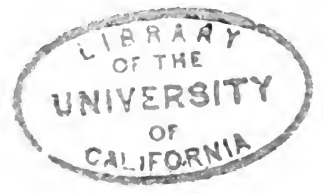

NEW YORK

JOHN WILEY \& SONS

LONDON: CHAPMAN \& HALL, Limited

I907 


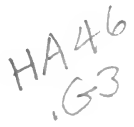

\section{CMIERAL'}

Copyright, 1906

BY

HENRY GANNETT 


\section{PREFACE.}

Statistics relating to the different peoples of the earth, their products of the soil, mines, and fisheries, their manufactures, means of transportation, and their commerce, are accessible, but in widely scattered form. To obtain them requires the consultation of many books, more than are commonly found in a single library. To bring these statistics together in compact form, thus making them easily accessible, is the purpose of this little book.

The figures given are the latest obtainable. Their date is not given, and it seems scarcely necessary. The dates of those in different tables differ in many cases, and even those relating to different countries in the same table may differ. This is unavoidable; otherwise the tables would be much less complete than they are.

Statistics of the most recent single year are given in preference to the mean of several years, as being on the whole nearest to the truth. Population, production, and commerce are increasing year by year, so that the mean of the five years 1900 to 1905 would be farther from the facts in 1906 than would be the figures of the single year 1905 .

The figures have been drawn from many' sources. To recapitulate them would be a useless extension of the work. 



\section{TABLE OF CONTENTS.}

PAGE

Area and Population by Continents........ I

Proportion of the Population of Each Race. I Proportion of the Population Speaking Certain Languages................ I Area and Population by Countries......... 2 Population of the Largest Cities......... 4 Average Rates of Increase of Population DURing the Last Half of the Nineteenth Century..................... I I

Proportion of EACH SEX............. II Proportion of ForEIGN-BORN............ I 2 PROPORTION OF THE FOREIGN-BORN OF EACH Leading Nationality.............. I 3 Proportion of the Population who are literate And Attend School. .............. I 3 Conjugal Condition................ I4 Birth, Death, and Marriage Rates........ I 5 Illegitimate BiRThs. . . . . . . . . . . . . . I6 Religious Adherents................. i6 Defectives................... I 7

Breadwinners, Total and By Sex......... I 7 Convictions for Homicide............. I 8 Breadwinners by Occupations........... I 8 Revenue, Expenditure, Debt, and Interest... I I9 Railway Mileage.................. 20 Tonnage of Merchant SHipping........... 2 I Production of Aluminum..............22 Production of Antimony...............22 Production of Arsenic................ 22 
PAGP

Production of Graphite............. 22

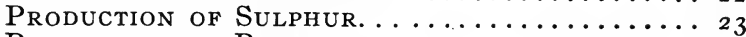

Production of Borax................ 3

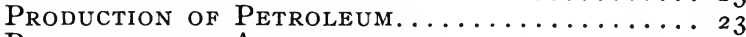

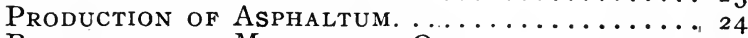

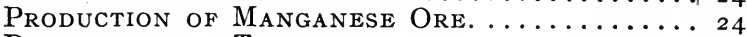

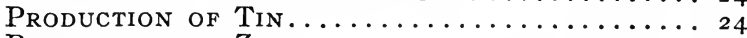

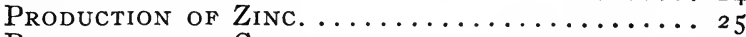

Production of Coal. .............. 25

Production of Copper................ 26

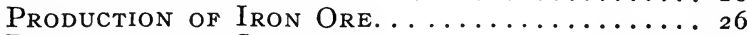

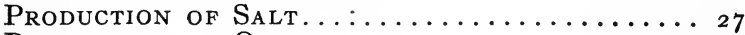

Production of Quicksilvier............ 27

Production of Pig Iron and Steel.......... 27

Production of Gold and Silver.......... 28

Consumption of Copper.............. 29

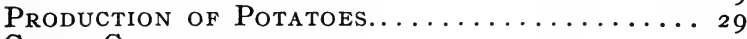

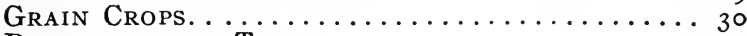

Production of Tobacco.................... 3 I

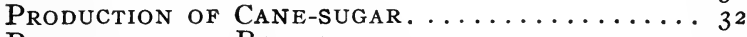

Production of Beet-sugar................ 32

Consumption of Sugar Per Capita................ 33

Importation of Sugar to the United States... 33

Production and Consumption of Coffee..... 34

Production and Consumption of Tea......34, 35

Production and Consumption of Cocoa....... 35

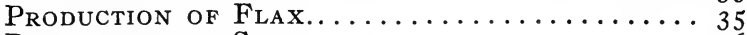

Production of Silk. . .............. 36

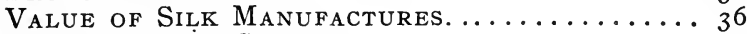

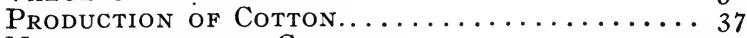

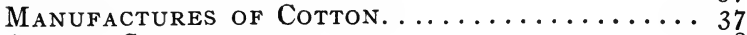

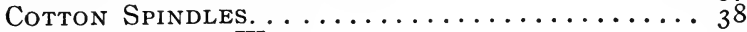

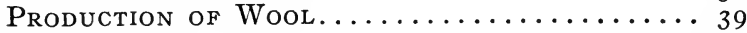

Lrve Stock...................... 40

Fisheries..................... 4 I

Production of Wine................ 4 I

Consumption of Liquors. . . . . . . . . . 42

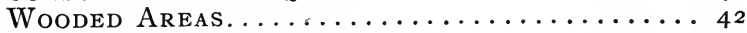

Cultivated Areas.................. 43

Foreign Commerce-Exports and IMPORTS.... 44 
Imports and Exports by Countries. ...... 45

Algeria...................... 45

Argentina. . . . . . . . . . . . . . .

Australia...................... 46

Austria-Hungary. ................. 46

Belgium....................... 47

Bulgaria.................... 48

Canada............................ 49

Cape Colony. .................... 49

Ceylon. ..................... 49

Chile...................... 49

China........................ $5^{\circ}$

Denmark. ........................ $5^{\circ}$

Dutch East Indies. . . . . . . . . . . .

Egypt........................

France..................

Germany.................... $5^{2}$

Greece. . . . . . . . . . . . . . .

India. . . . . . . . . . . . . . . . 54

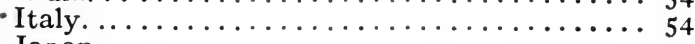

Japan...................... 55

Mexico....................... 55

Natal. .................... 56

Netherlands. . . . . . . . . . . . . . 56

New Zealand................... 57

Norway. ................... 57

Philippine Islands. . . . . . . . . . . . 57

Portugal......................... $5^{8}$

Rumania........................ 58

Russia......................... 58

Servia...................... 59

Siam. ......................... 59

Spain. ...................... 59

Straits Settlements. . . . . . . . . . . 60

Sweden........................60

Switzerland. ..................60

Tunis. .................... 6 r

Turkey. ................. 6r

United Kingdom. ................. $6_{2}$

United States................... 63

Uruguay. ...................... 64 
Imports AND Exports By Commodities........ 65 Argentina........................... 65

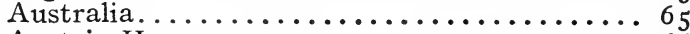

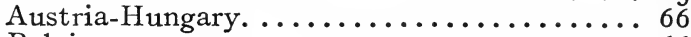
Belgium.....................666

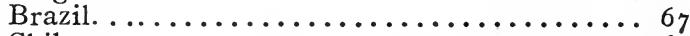

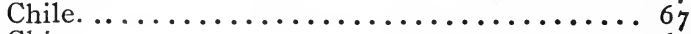

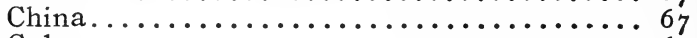

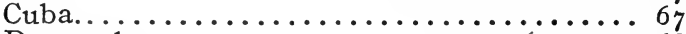

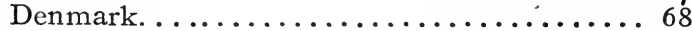

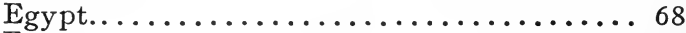

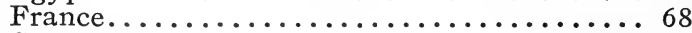

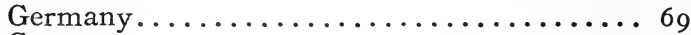

Greece. .....................6 69

India. ........................ 69

Italy.......................

Japan....................... 70

Mexico ....................... 70

Netherlands....................... 7 I

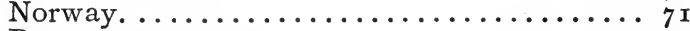

Peru......................... 7 I

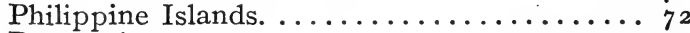

Portugal..........................

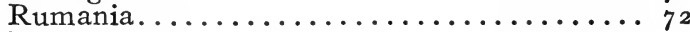

Russia.......................... $7_{2}$

Servia....................... 73

Spain. ........................ 73

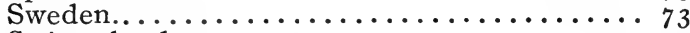

Switzerland................... 74

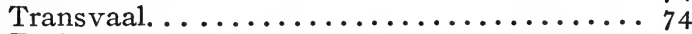

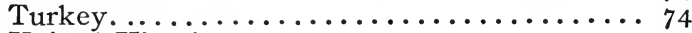

United Kingdom................ 75

United States...................... $7^{6}$ Uruguay.......................... $7^{6}$ 


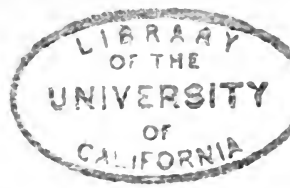

\section{STATISTICAL ABSTRACT OF THE WORLD.}

\section{AREA AND POPULATION.}

By Continents.

\begin{tabular}{|c|c|c|c|}
\hline & $\begin{array}{c}\text { Area } \\
\text { Square Miles. } \\
\text { Tens of } \\
\text { Thousands. }\end{array}$ & $\begin{array}{l}\text { Population. } \\
\text { Thousands. }\end{array}$ & $\begin{array}{l}\text { Inhabitants } \\
\text { Per } \\
\text { Square Mile. }\end{array}$ \\
\hline Africa. . .......... & I I , 403 & 140,700 & I 3 \\
\hline Asia. ............ & I 5,085 & 8 i $9,55^{6}$ & 57 \\
\hline Europe. . . . . . . . & 3,796 & 392,264 & 104 \\
\hline North America..... & 8,559 & 105,714 & I 3 \\
\hline 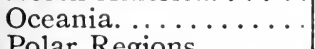 & 4,2 I 5 & 6,483 & I \\
\hline $\begin{array}{l}\text { Polar Regions. . . . . . } \\
\text { South America....... }\end{array}$ & 7,508 & $\begin{array}{r}91 \\
38,482\end{array}$ & 5 \\
\hline World. . & 50.656 & & \\
\hline woike. & 50,050 & $1,503,290$ & 30 \\
\hline
\end{tabular}

PROPORTION OF THE POPULATION OF EACH RACE.

White.......... 4 I

Yellow............. 45

Black........... I I

Brown........... 2

Red............ I
|PROPORTION OF THE POPULATION SPEAKING CERTAIN LANGUAGES.

English......... Io

French.......... 3

German................ 5

Italian.......... 2

Portuguese........ I

Russian......... 7

Spanish......... 3 


\section{AREA AND POPULATION.}

By Countries.

\begin{tabular}{|c|c|c|c|}
\hline & $\begin{array}{c}\text { Area, } \\
\text { Square Miles. }\end{array}$ & Population. & $\begin{array}{l}\text { Density of } \\
\text { Population }\end{array}$ \\
\hline Abyssinia. ....... & 312,000 & $8,330,000$ & 27 \\
\hline Afganistan. ...... & 241,000 & $4,55^{\circ}, 000$ & I9 \\
\hline Alaska.......... & 591,000 & 64,000 & \\
\hline Algeria.......... & 343,600 & $4,800,000$ & I 4 \\
\hline Arabia..... & 880,000 & $95^{\circ}, 000$ & $\mathrm{I}$ \\
\hline Argentina. . . . . . & $I, I 00,000$ & $5,000,000$ & 5 \\
\hline Australia.......... & $2,973,000$ & $4,000,000$ & $\mathrm{I} \frac{1}{2}$ \\
\hline Austria-Hungary . & 201,300 & 47, I 50,000 & $180^{\circ}$ \\
\hline Baluchistan...... & I $4 \mathrm{I}, 600$ & 742,000 & 5 \\
\hline Belgium. . . . . . . & I I, 400 & $6,700,000$ & 587 \\
\hline Bolivia... & 443,000 & $\mathrm{r}, 776,000$ & 4 \\
\hline Brazil. ....... & $3,300,000$ & $14,334,000$ & $4 \frac{1}{2}$ \\
\hline British Borneo. ... & 88,000 & 800,000 & 9 \\
\hline British S. Africa... & I $, 197,000$ & $7,000,000$ & 6 \\
\hline British W. Indies.. & I 2,000 & $\mathrm{I}, 65^{\circ}, 000$ & I 37 \\
\hline Bulgaria......... & 37,300 & $3,750,000$ & 100 \\
\hline Canada.......... & $3,756,000$ & $5,375,000$ & $\mathrm{I} \frac{1}{2}$ \\
\hline Central America. . & 206,000 & $4, \mathrm{I} 36,000$ & 20 \\
\hline Ceylon. . . . . . . . & 25,000 & $3,600,000$ & I 44 \\
\hline Chile. . . . . . & 293,000 & $3,175,000$ & I I \\
\hline China proper. . & $I, 500,000$ & $407,300,000$ & 272 \\
\hline Colombia. . . . & 466,000 & $3,917,000$ & 8 \\
\hline Cuba..... & 44,000 & $\mathrm{I}, 570,000$ & 36 \\
\hline Denmark. ....... & I 4,850 & $2,450,000$ & 165 \\
\hline Dutch East Indies & $5^{85}, 000$ & $35,000,000$ & 60 \\
\hline Ecuador. . ........ & I I 8,600 & I, 300,000 & Io \\
\hline Egypt proper. .... & 248,000 & $9,700,000$ & 39 \\
\hline France.......... & 207,000 & $39,000,000$ & I 88 \\
\hline French Indo-China & $25^{6,000}$ & I $5,600,000$ & $6 \mathrm{I}$ \\
\hline Germany......... & 210,000 & $5^{6,400,000}$ & 269 \\
\hline Greece. ....... & 25,000 & $2,435,000$ & 97 \\
\hline Greenland. .. . & 838,000 & I 2,000 & \\
\hline Guianas..... & I 75,000 & 4 I 5,000 & $2 \frac{1}{2}$ \\
\hline Haiti ... & II, 000 & I $, 294,000$ & I I 8 \\
\hline Hawaii. . . . . . . . . & $6,4,50$ & I 54,000 & 24 \\
\hline
\end{tabular}




\section{AREA AND POPULATION-Continued.}

\begin{tabular}{|c|c|c|c|}
\hline & $\begin{array}{c}\text { Area, } \\
\text { Square Miles. }\end{array}$ & Population. & $\begin{array}{l}\text { Density of } \\
\text { Population }\end{array}$ \\
\hline Iceland......... & 40,000 & 80,000 & 2 \\
\hline 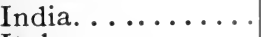 & I ,790,000 & $298,000,000$ & I 67 \\
\hline 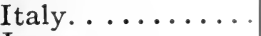 & I I 0,700 & $32,500,000$ & 294 \\
\hline 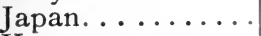 & I 6 I, I 50 & $46,500,000$ & 289 \\
\hline 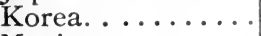 & 84,250 & $9,670,000$ & I I 5 \\
\hline Mexico......... & 767,000 & I $3,600,000$ & I 8 \\
\hline Morocco........ & 176,000 & $7,000,000$ & 40 \\
\hline Netherlands..... & I 2,500 & $5,100,000$ & 408 \\
\hline Newfoundland.... & 42,700 & 217,000 & 5 \\
\hline New Guinea..... & 311,000 & 700,000 & 2 \\
\hline New Zealand. ... & 104,600 & 8 I 6,000 & 8 \\
\hline Norway......... & I 24,000 & $2,220,000$ & I 8 \\
\hline Paraguay....... & 97,700 & 636,000 & $6 \frac{1}{2}$ \\
\hline 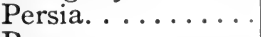 & 635,000 & $9,000,000$ & 14 \\
\hline 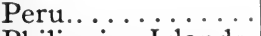 & 439,000 & $4,586,000$ & Io \\
\hline Philippine Islands & I I 5,000 & $7,636,000$ & 66 \\
\hline Porto Rico....... & 3,600 & 953,000 & 265 \\
\hline Portugal........ & 35,700 & $5,400,000$ & I $5 \mathrm{I}$ \\
\hline Rumania......... & 50,700 & $5,900,000$ & I I 6 \\
\hline Russia in Asia.... & $6,672,000$ & $21,200,000$ & 3 \\
\hline Russia in Europe. & 2 , I I 3,000 & $109,700,000$ & $5^{2}$ \\
\hline Santo Domingo... & I 8,700 & 416,000 & 22 \\
\hline 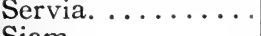 & I 8,600 & $2,500,000$ & I 34 \\
\hline 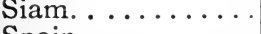 & 244,800 & $6,320,000$ & 26 \\
\hline 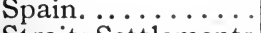 & I95,000 & I $8,500,000$ & 95 \\
\hline Straits Settlements & 35,500 & $\mathrm{I}, 45^{2}, 000$ & $4 I$ \\
\hline 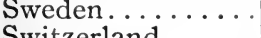 & I 73,000 & $5,100,000$ & 30 \\
\hline Switzerland..... & I 6,000 & $3,325,000$ & 208 \\
\hline 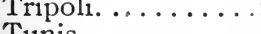 & 405,800 & $1,000,000$ & $2 \frac{1}{2}$ \\
\hline Tunis. $\cdots \cdots$ & 64,600 & $\mathrm{I}, 800,000$ & 28 \\
\hline Turkey in Asia.... & 680,000 & I 7,200,000 & 25 \\
\hline Turkey in Europe. & 64,600 & $5,000,000$ & $9 I$ \\
\hline United Kingdom. & I 2 I , 000 & $42,000,000$ & 347 \\
\hline United States..... & $3,026,500$ & $76,600,000$ & 25 \\
\hline Uruguay....... & 60,000 & 978,000 & I 4 \\
\hline venezuela....... & .364 .000 & $2,445,000$ & 7 \\
\hline
\end{tabular}




\section{POPULATION OF THE LARGEST CITIES.}

Aachen, Germany.

Aberdeen, Scotland

I 35,245

Agra, India.

Ahmadabad, India.

I 43,722

I 88,022

Aleppo, Turkey.

Alexandria, Egypt.

Algiers, Algeria.

Allahabad, India.

I 85,889

I 27, I 50

319,766

I 38,708

Allegheny, U. S.

I 72,032

I 38,0 , 8

Altona, Germany.

I 61,501

Amoy, China

Amritsar, India.

I I 3,800

162,429

Amsterdam, Netherlands.............

Antwerp, Belgium

546,534

286,695

Astrakan, Russia.

I 2 I, 580

Athens, Greece.

I I I 486

Bagdad, Turkey.

145,000

Bahia, Brazil

Bale, Switzerland.

Baltimore, U, S

I 74,4 I 2

I 20,897

Bangalore, India

$53 \mathrm{I}, 3 \mathrm{I} 3$

Barcelona, Spain.

I 59,046

Bareilli, India.

Barmen, Germany.

533,000

I 3 I, 208

I $4 \mathrm{I}, 944$

Baroda, India.

Batavia, Java.

I 03,790

I I 5,887

Beirut, Turkey

Belfast, Ireland. . . . . . . . . . . . .

Benares, India.

I 18,800

349,180

$209,33 \mathrm{I}$

Berlin, Germany.

Birkenhead, England.

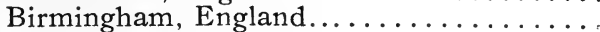

Blackburn, England.

Bologne, Italy.

Bolton, England.

Bombay, India.

Bordeaux, France.

Boston, U. S.

Bradford, England.

Bremen, Germany.

$\mathrm{I}, 888,848$

I I 0,9 I 5

522,204

I 27,626

I 52,009

I 68,2 I 5

776,006

257,638

595,380

279,767

I63,297 


\section{POPULATION OF THE LARGEST CITIES-Contd.}

Breslau, Germany.

Brighton, England. ................

Bristol, England.

Brunn, Austria.

Brunswick, Germany

Brussels, Belgium.

Bucharest, Rumania

Budapest, Hungary.

422,709

I 23,478

328,945

109,346

128,226

586,936

276, I 78

732,322

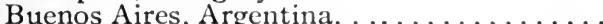

Buffalo, U. S.

Byelaya Tserkof, Russia...............

Cairo, Egypt.

Calcutta, India.

Canton, China.

Cardiff, England.

891,268

376,587

I 20,700

570,062

$1,026,987$

880,000

Catania, Italy.

164,333

I 49,295

197,170

189,305

206,9 I 3

Charlottenburg, Germany..............

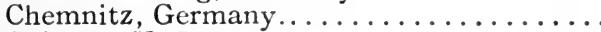

Chicago, U. S.

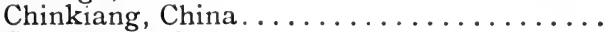

Chungking, China.

$1,873,880$

160,000

350,000

Cincinnati, U. S .

$33^{2}, 934$

Cleveland, U.S.

4 I $4,95^{\circ}$

Cologne, Germany. . . . . . . . . . . . . . .

372,529

Colombo, Ceylon.

158,228

Columbus, U. S.

I 35,487

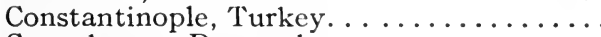

Copenhagen, Denmark.

I , I 2 5,000

Croydon, England .

378,235

Damascus, Turkey.

I 33,895

225,000

Dantzig, Germany

Delhi, India.

Denver, U. S .

I 40,563

208,575

I 33,859

I05,91 2

Derby, England.

317,591

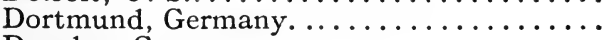

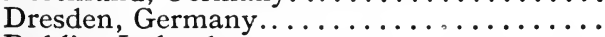

Dublin, Ireland.

I 42,733

396, I 46

290,638 


\section{POPULATION OF THE LARGEST CITIES-Contd.}

Dundee, Scotland...................

Düsseldorf, Germany. .

I $60,87 \mathrm{I}$

Edinburgh, Scotland.

2 I 3,7 I I

Ekaterinoslaf, Russia.

316,479

Elberfeld, Germany.

I $35,55^{2}$

Essen, Germany

I 56,966

Fall River, U. S.

I I 8,862

Fez, Morocco.

Florence, Italy.

Frankfort-on-Main, Germany. . . . . . . .

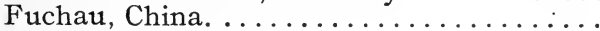

Gateshead, England. . . . . . . . . . . . 105,762 140,000 205,589 288,989 624,000 I09,888 Geneva, Switzerland

Genoa, Italy.

Ghent, Belgium.

Glasgow, Scotland.

I I 0,954

234,7 I0 I 62,925

Göteborg, Sweden.

735,906

Gratz, Austria.

I 35,3 I 4

I 38,080

Guadalajara, Mexico.

I OI, 208

Hague, Netherlands.

229,839

Halifax, England.

Halle-on-Saal, Germany............... I04,936 I 56,609

Hamburg, Germany. $705,73^{8}$

Hangchau, China.

Hankau, China.

700,000

870,000

Hanoi, Tonking.

I 50,000

Hanover, Germany.

235,649

275,000

Havre, France.

I 30,196 I 00,8 I 2

Helsingfors, Russia. ...............

Hiroshima, Japan.

I 2 I, I 96

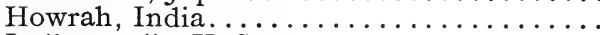

Indianapolis, U. S.

I 57,594

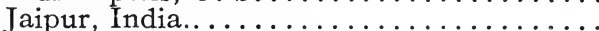

I 9 I, 033 160,167

Jersey City, U. S

Kansas City, U.S.

232,699

I 73,064

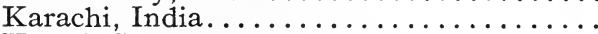

Kassel, Germany.

I 16, I 63 106,034

Kazan, Russia. .

$143,7 \circ 7$ 


\section{POPULATION OF THE LARGEST CITIES-Contd.}

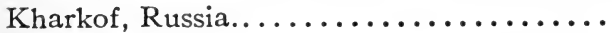
Kiel, Germany. Kiev, Russia.

Kingston-upon-Hull, England. Kisheneff, Russia.

Kobe, Japan.

Königsberg, Germany.............

Krefeld, Germany.

Kristiania, Norway.

197,405

107,977

319,000

240,259

I 25,787

285,002

I 89,483

106,893

Kyoto, Japan.

Lahore, India.

227,626

380,568

Leeds, England

202,964

Leicester, England.

428,968

Leipzig, Germany.

2 I I 579

Lemberg, Austria

$45^{6, \text { I } 24}$

Liege, Belgium.

I 59,877

I 66,105

Lille, France.

210,696

Lima, Peru.

Lisbon, Portugal.

Liverpool, England

I 30,000

356,009

Lodz, Russia.

$684,95^{8}$

London, England.

Los Angeles, U. S.

$35 \mathrm{I}, 57^{\circ}$

Louisville, U. S. .

$6,5^{81}, 37^{2}$

I 16,420

Lucknow, India.

215,402

264,049

Lyon, France.

459,099

Madras, India

509,346

Madrid, Spain

539,835

Madura, India.

I0 5,984

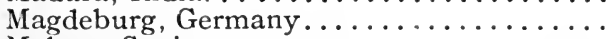

229,667

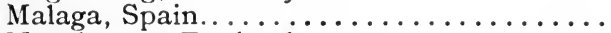

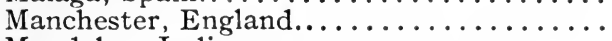

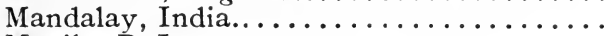

I 30,109

Manila, P. I. . . . . . . . . . . . .

543,872

I 83,8 I 6

Mannheim, Germany.

Marseilles, France.

Meerut, India.

219,928

I 4 I, I 3 I

$49 \mathrm{I}, \mathrm{I} 6 \mathrm{I}$

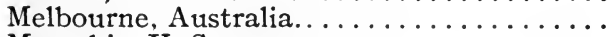
Memphis, U. S.

I I 8 , 129 501,460 I $13,6 \times 9$ 


\section{POPULATION OF THE LARGEST CITIES-Contd.}

Messina, Italy.................

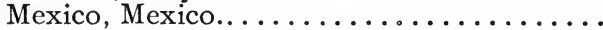

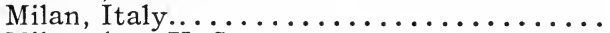

Nilwaukee, U. S

Minneapolis, U.S.

Montevideo, Uruguay.

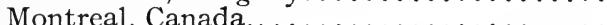

Moscow, Russia.

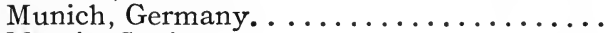

Murcia, Spain.

Nagasaki, Japan...................

Nagoya, Japan

Nagpur, India.

Nancy, France.

Nanking, China.

Nantes, France.

Naples, Italy

Newark, U.S.

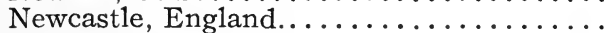

New Haven, U. S.

New Orleans, U.S

New York, U. S.

Nice, France.

149,778

344,721

$49 \mathrm{I}, 460$

312,948

$26 \mathrm{r}, 974$

276,034

267,730

$\mathrm{I}, 092,360$

$499,93^{2}$

I I I 539

I 53,293

288,639

I 77,734

IO2, 559

250,000

r 32,990

563,540

283,289

215,328

I I 4,600

300,625 $4,013,781$

I05, 109

Ningpo, China.

260,000

Norwich, England.

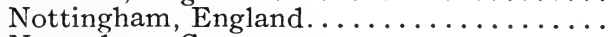

Nuremberg, Germany.

I I I, 753

239,743

26 I, 08 I

Odessa, Russia

Oldham, England..

449,673

I 37,246

Omaha, U. S.

Oporto, Portugal.

I I 3,36 I

I 67,955

Osaka, Japan. ..................

Palermo, Italy.

995,945

309,694

Paris, France.

Paterson, U. S

Patna, India.

Pernambuco, Brazil.

$2,714,068$

I I I , 529

I 34,785

I I $\mathrm{X}, 556$

Philadelphia, U. S

Pittsburg, U. S

$\mathrm{I}, 367,7 \mathrm{I} 6$

345,043 


\section{POPULATION OF THE LARGEST CITIES-Contd.}

Plymouth, England.

Poona, India.

107,636

Portsmouth, England.

I 53,320

Posen, Germany..

I 80,133

Prague, Austria.

I 17,033

Preston, England.

$201,5 \times 9$

I 12,989

Providence, U. S.

Rangoon, India.

I 98,635

Reims, France.

234,88 I

Riga, Russia. .

108,385

Rio Janeiro, Brazil.

Rochester, U. S.

Rome, Italy. .

Rostof-on-Don, Russia

256,197

$522,65 \mathrm{I}$

I 81,666

462,783

Rotterdam, Netherlands

I 19,889

Roubaix, France.

Rouen, France.

357,474

142,365

St. Etienne, France.

I 6,316

146,559

St. Joseph, U. S. .

I 10,479

St. Louis, U. S.

612,279

St. Paul, U. S.

St. Petersburg, Russia.

197,023

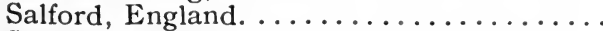

Salonica, Turkey.

1,3 r 3,300

220,957

105,000

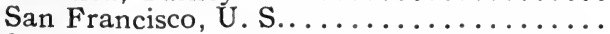

Santiago, Chile.

355,9 I 9

296,695

Saratof, Russia. .

143,43 I

Scranton, U.S.

107,026

Sendai, Japan.

Seoul, Korea.

100,231

Seville, Spain.

I 96,646

I 48,3 I 5

Shanghai, China

620,000

Sheffield, England.

380,793

Smyrna, Turkey.

201,000

Soerabaya, Java.

I 46,944

Southampton, England.

I04,824

Srinagar, India.

122,6 I 8

Stettin, Germany.

210,702

Stockholm, Sweden

3 II, 043 


\section{POPULATION OF THE LARGEST CITIES-Contd.}

Strassburg, Germany...............

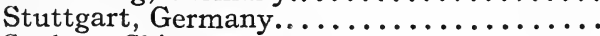

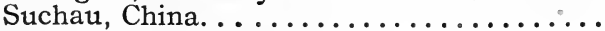

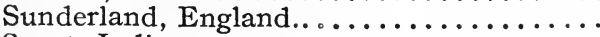
Surat, India.

Syracuse, U. S. Szeged, Hungary Tabriz, Persia. Teheran, Persia Tientsin, China. Tokyo, Japan. Toledo, U.S. Torino, Italy. Toronto, Canada Toulon, France. Toulouse, France.

Trichinopoli, India................ Trieste, Austria.

Tula, Russia.

Tunis, Tunis.

Utrecht, Netherlands.

Valencia, Spain.

Valparaiso, Chile.

Venice, Italy.

Victoria, Hongkong.

Vienna, Austria.

Vilna, Russia.

Warsaw, Russia.

Washington, U. S.

Westham, England

Worcester, U.S.

Wuhu, China.

Yokohama, Japan.

Zurich, Switzerland.
I 5 I, O4I

г 6,699 500,000 I 46,077 I I 9,306 I 7,503 I०2,99I 200,000 280,000 750,000 I $, 8 \mathrm{I} 8,655$ I 45,9 O I 335,656 208,040 I02,602 I 49,84 I I $04,72 \mathrm{I}$ I 34, I 43 IO9, $35^{2}$ I 70,000 I I0, 648 213,530 I 32,94 I I 5 I, 840 283,905 $I, 674,957$ I 62,633 756,426 $293,2 \times 7$ $267,35^{8}$ $\mathrm{x} 28, \mathrm{I} 35$ II 5,580 326,035 I 69,4IO 


\section{AVERAGE RATES OF INCREASE OF POPULATION}

During Last Half of the Nineteenth Century.

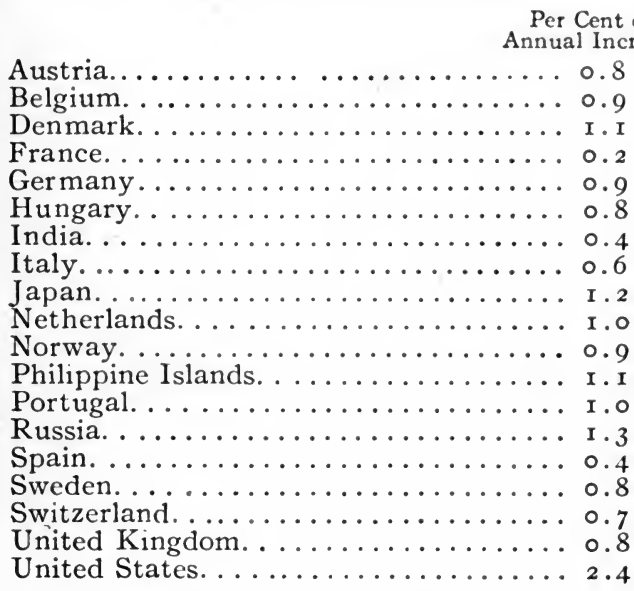

\section{PROPORTION OF EACH SEX.}

\begin{tabular}{|c|c|c|}
\hline & $\begin{array}{l}\text { Per } \\
\text { Males. }\end{array}$ & $\begin{array}{l}\text { ent of } \\
\text { Females }\end{array}$ \\
\hline Aro & 52.8 & 47.2 \\
\hline & $49 . \mathrm{I}$ & 50.9 \\
\hline elgium............ & $49 \cdot 7$ & \\
\hline$\ldots \ldots \ldots \ldots \ldots \ldots \ldots \ldots \ldots$ & $5 \mathrm{I} .2$ & 48.8 \\
\hline & 53.0 & 47.0 \\
\hline$\ldots \ldots \ldots \ldots \ldots$ & $5 \mathrm{I} \cdot 8$ & \\
\hline$\cdots \ldots \ldots \ldots \ldots \ldots \ldots \ldots$ & $48 \cdot 7$ & $5 \mathrm{I} \cdot 3$ \\
\hline$\ldots \ldots \ldots \ldots$ & $\begin{array}{l}49 \cdot 4 \\
49.2\end{array}$ & $\begin{array}{l}50.0 \\
50.8\end{array}$ \\
\hline & 50.9 & 49.1 \\
\hline & 47.8 & 52.2 \\
\hline
\end{tabular}




\section{PROPORTION OF EACH SEX-Continued.}

\begin{tabular}{|c|c|c|}
\hline \multirow[b]{2}{*}{ Japan. ................ } & \multicolumn{2}{|c|}{ Per Cent of } \\
\hline & 50.4 & 49.6 \\
\hline & 49.0 & $5 \mathrm{I} .0$ \\
\hline Mexico...... & 49.6 & $50 \cdot 4$ \\
\hline Netherlands...... & $49 \cdot 4$ & 50.6 \\
\hline Philipnine Islands. $\ldots \ldots \ldots \ldots \ldots$ & 50.0 & 50.0 \\
\hline Porto Rico. . . . . . . . . . & $49 \cdot 5$ & 50.5 \\
\hline Portugal. . . . . . . & 47.8 & 52.2 \\
\hline Spain. $\ldots \ldots \ldots \ldots \ldots \ldots \ldots \ldots$ & $48 \cdot 5$ & $5 \mathrm{I} \cdot 5$ \\
\hline Straits Settlements. . . . . . . . . . . & $67 \cdot 3$ & $32 \cdot 7$ \\
\hline Switzerland. $\ldots \ldots \ldots \ldots \ldots \ldots \ldots$ & 48.6 & 5 I 4 \\
\hline United Kingdom. $\ldots \ldots \ldots \ldots \ldots \ldots$ & 48.5 & $5 \mathrm{I} \cdot 5$ \\
\hline United States. . . . . . . . . . . . . & $5 \mathrm{I} . \mathrm{I}$ & 48.9 \\
\hline
\end{tabular}

\section{PROPORTION OF FOREIGN-BORN POPULATION.}

Per Cent.

Argentina.................... I 7

Austria-Hungary................ I.6

Belgium.................. 3. I

Canada..................... I 3.0

Chile..................... 2.3

Cuba...................... II.

Denmark.................... $3 \cdot 3$

Egypt.................... I 2

France.................... 2.7

Germany................... I.5

Greece.................... I.3

Italy ...................... 0.2

Mexico...................... 0.4

Netherlands................. I 0

Norway..................... 4.0

Philippine Islands. . . . . . . . . . . 0.8

Portugal.................... 0.8

Rumania..................... 3.I

Servia................... I.0

Sweden..................... 0.5

Switzerland................. II.8

United States................ 1 3.6 
PERCENTAGE WHICH THE FOREIGN-BORN OF EACH LEADING NATIONALITY BEAR TO THE TOTAL FOREIGN-BORN.

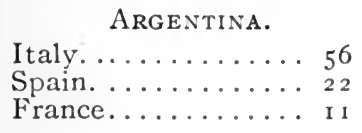

CANADA.

United Kingdom. ... 55 United States...... I Germany........ 4 Russia. ........ 5

\section{Chile.}

Germany......... Io Spain........... I I France.......... I I United Kingdom.... 9 Italy............ Io Argentina........ Io
Cuba.

Spain.......... 75

Africa.......... 7

United States...... 4

MEX co.

Spain.......... 28

United States...... 27

Guatemala........ Io

UN.T.D STAT_L.

Germany............. 26

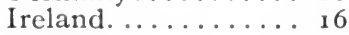

England......... 8

Canada................ I

Sweden................... 6

Italy.......... 5

Russia......... 4

PROPORTION OF THE POPULATION WHO ARE LITERATE AND WHO ATTEND SCHOOL.

Literate. Attend School.

Australia................

Austria-Hungary. ......... 55

20

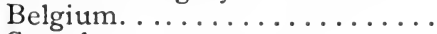

55

Canada.

$2 \mathrm{I}$

Cuba................... 34

- 6

Denmark

France.

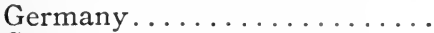

Greece.

India. ............... 5

Italy.

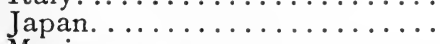

Mexico.

Netherlands 


\section{PROPORTION OF THE POPULATION WHO ARE LITERATE AND WHO ATTEND SCHOOL-Contd.}

Literate. Attend School.

New Zealand..............

Norway. ...................

Philippine Islands. . . . . . . I 4

Porto Rico. .............. I 5

Portugal.

I 7

I 5

Rumania...............22

Russia. ................

Servia................ 7

Spain................. 29

Sweden............... I4

Switzerland............. 20

United Kingdom. . . . . . . I8

United States............ $68 \quad 22$

CONJUGAL CONDITION.

\begin{tabular}{|c|c|c|c|}
\hline \multirow[b]{2}{*}{, } & \multicolumn{3}{|c|}{ Proportion of } \\
\hline & Single & Married. & Widowed. \\
\hline 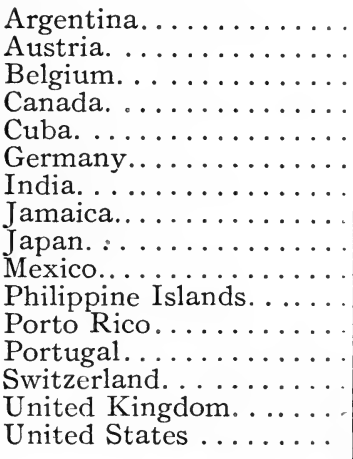 & $\begin{array}{l}69 \cdot 7 \\
60.1 \\
60 \cdot 0 \\
61 \cdot 7 \\
70 \cdot 5 \\
59 \cdot 5 \\
41 \cdot 9 \\
77 \cdot 5 \\
62.0 \\
56 \cdot 4 \\
69 \cdot 7 \\
60 \cdot 7 \\
61.1 \\
62.0 \\
57 \cdot 9\end{array}$ & $\begin{array}{l}26 \cdot 0 \\
34 \cdot 3 \\
34 \cdot 2 \\
34 \cdot I \\
24 \cdot I \\
34 \cdot 8 \\
46 \cdot 5 \\
18 \cdot 6 \\
35 \cdot 6 \\
31 \cdot 0 \\
36 \cdot 4 \\
25 \cdot 4 \\
33 \cdot I \\
32 \cdot I \\
32 \cdot 3 \\
36 \cdot 5\end{array}$ & $\begin{array}{r}4 \cdot 3 \\
5 \cdot 6 \\
5 \cdot 8 \\
4 \cdot 2 \\
5 \cdot 4 \\
5 \cdot 7 \\
I 1 \cdot 6 \\
3 \cdot 9 \\
7 \cdot 0 \\
7 \cdot 2 \\
4 \cdot 9 \\
6 \cdot 2 \\
6.8 \\
5 \cdot 7 \\
5 \cdot 1\end{array}$ \\
\hline
\end{tabular}


STATISTICAL ABSTRACT OF THE WORLD. I5

\section{BIRTH, DEATH, AND MARRIAGE RATES.}

Australia.............

Austria.

Belgium.

Cape Colony. ..............

Ceylon.

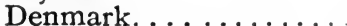

France................

Germany.............

Hungary

India.

Italy.

Japan.

Netherlands.

New Zealand

Norway. ...

Philippine Islands. . .....

Portugal.

Rumania.

Russia.

Servia.

\begin{tabular}{|c|c|c}
$\begin{array}{c}\text { Birth- } \\
\text { rate. }\end{array}$ & $\begin{array}{c}\text { Death- } \\
\text { rate. }\end{array}$ & $\begin{array}{c}\text { Marriage- } \\
\text { rate. }\end{array}$ \\
\cline { 1 - 3 } 27.0 & I3.0 & 7.0 \\
36.6 & 24.0 & 8.1 \\
29.4 & 17.1 & 8.4 \\
41.9 & 22.3 & 8.2 \\
34.0 & 16.0 & \\
34.3 & 27.6 & \\
29.8 & 15.8 & 7.2 \\
22.0 & 20.1 & 7.8 \\
35.7 & 20.7 & 8.2 \\
37.8 & 25.4 & 8.8 \\
37.4 & 36.8 & \\
32.5 & 21.9 & 7.2 \\
31.4 & 20.3 & 7.7 \\
32.3 & 17.2 & 7.7 \\
26.0 & 10.0 & 8.0 \\
29.8 & 14.9 & 6.7 \\
47.9 & 31.7 & 10.0 \\
30.1 & 20.3 & 6.7 \\
39.5 & 26.3 & 7.2 \\
49.5 & 32.8 & 9.2 \\
38.0 & 21.0 & 8.3 \\
34.4 & 29.4 & 8.8 \\
26.8 & 16.0 & 6.0 \\
29.1 & 18.0 & 7.6 \\
28.2 & 18.4 & \\
35.1 & 17.4 & \\
& & \\
& &
\end{tabular}

Spain.

Sweden

Switzerland

United Kingdom. 


\section{ILLEGITIMATE BIRTHS.}

Percentage of All Births.

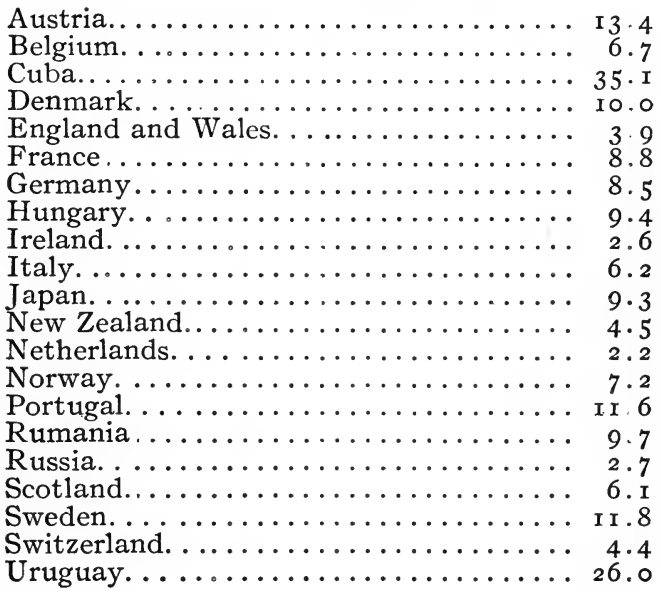

\section{RELIGIOUS ADHERENTS.}

In MiLLIONS.

Animists. . . . . . . . . . . . . ${ }^{5} 8$

Buddhists. . . . . . . . . . . . . I 38

Confucianists................. 292

Greek Church................. II II

Hindus...................... 2 ro

Jews....................... Io

Mohammedans................. 2 I 2

Protestants................... I 78

Roman Catholics................ 262

Shintoists.................. 25 


\section{DEFECTIVES.}

Per Million of Population.

\begin{tabular}{|c|c|c|c|c|}
\hline & Blind. & $\begin{array}{l}\text { Deaf- } \\
\text { mutes. }\end{array}$ & $\begin{array}{l}\text { Insane } \\
\text { and } \\
\text { Idiots. }\end{array}$ & Total. \\
\hline Austria.......... & 806 & 1292 & 2169 & 4267 \\
\hline England and Wales. & 809 & 489 & 3357 & 4655 \\
\hline Hungary. . . . . . . . & 1051 & 1089 & 1612 & $375^{2}$ \\
\hline Ireland..... & I1 35 & 715 & $45 \circ 3$ & 6353 \\
\hline Norway: & 1289 & 1080 & 3896 & 6265 \\
\hline Scotland...... & 695 & $5^{28}$ & 3840 & 5063 \\
\hline Sweden.......... & 825 & 1019 & 3410 & 5254 \\
\hline United States.... & 805 & 659 & 3223 & 4687 \\
\hline
\end{tabular}

\section{BREADWINNERS.}

Per Cent of Total of each Sex and of the Total Population.

Austria.

Belgium.

Cuba.

Denmark

France..

Germany.

Hungary

Italy.

Netherlands.

Norway.

Philippine Islands. ..........

Sweden...

Switzerland.

United Kingdom.

United States..

\begin{tabular}{|c|c|c} 
Males. & Females & All. \\
\hline 63 & 47 & 55 \\
60 & 26 & 43 \\
68 & 9 & 40 \\
58 & 21 & 39 \\
64 & 33 & 48 \\
6 I & 25 & 43 \\
63 & 25 & 44 \\
66 & 40 & 53 \\
59 & 17 & 38 \\
56 & 24 & 39 \\
58 & 29 & 44 \\
55 & 20 & 37 \\
6 I & 29 & 45 \\
63 & 27 & 45 \\
6 I & I 4 & 38 \\
& & \\
& &
\end{tabular}




\section{BREADWINNERS BY OCCUPATIONS.}

Per Cent of Total.

\begin{tabular}{|c|c|c|c|c|c|c|c|}
\hline ' & 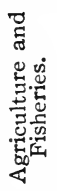 & 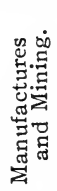 & 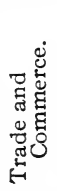 & 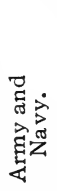 & 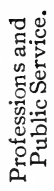 & 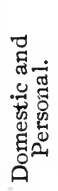 & $\begin{array}{c}\stackrel{\dot{0}}{0} \\
\stackrel{\mathrm{S}}{0}\end{array}$ \\
\hline 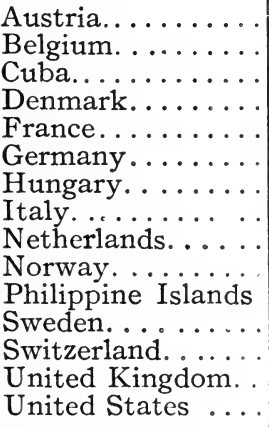 & $\begin{array}{l}64 \\
23 \\
48 \\
27 \\
44 \\
36 \\
59 \\
57 \\
31 \\
50 \\
41 \\
54 \\
37 \\
15 \\
36\end{array}$ & $\begin{array}{l}22 \\
38 \\
15 \\
24 \\
34 \\
37 \\
13 \\
28 \\
34 \\
23 \\
32 \\
15 \\
41 \\
54 \\
24\end{array}$ & $\begin{array}{r}6 \\
\text { I } 2 \\
\text { I } 3 \\
8 \\
9 \\
\text { I I } \\
3 \\
4 \\
\text { I } 7 \\
\text { I } 2 \\
7 \\
6 \\
\text { I I } \\
\text { I } 0 \\
\text { I } 6\end{array}$ & $\begin{array}{l}\text { I } \\
3 \\
3 \\
2 \\
I \\
I \\
2\end{array}$ & $\begin{array}{l}\text { I } \\
5 \\
5 \\
4 \\
2 \\
3 \\
5 \\
3 \\
I \\
3 \\
4 \\
6\end{array}$ & $\begin{array}{r}4 \\
25 \\
23 \\
26 \\
5 \\
6 \\
5 \\
4 \\
10 \\
10 \\
19 \\
14 \\
6 \\
14 \\
19\end{array}$ & $\begin{array}{r}9 \\
3 \\
16 \\
3 \\
2\end{array}$ \\
\hline
\end{tabular}

\section{CONVICTIONS FOR HOMICIDE.}

Per Million Inhabitants.

Austria................. 29

Belgium................ 23

England................ 8

France................. I9

Germany................ I4

Hungary. ................ 58

Ireland................. I4

Italy.................. I 20

Netherlands.............. 9

Scotland................ 7

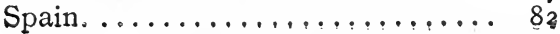




\section{REVENUE, EXPENDITURES, DEBT, AND INTEREST.}

In Millions of Dollars.

\begin{tabular}{|c|c|c|c|c|}
\hline & Revenue. & $\begin{array}{l}\text { Expendi- } \\
\text { ture. }\end{array}$ & Debt. & Interest. \\
\hline Algeria. & I0.9 & 10.5 & & \\
\hline Argentina. & 62.7 & 60.8 & 479.8 & 35.0 \\
\hline Austraila........... & 140.8 & 142.1 & 1084.6 & 42.0 \\
\hline Austria-Hungary. & $75 \cdot 9$ & $75 \cdot 9$ & 11075 & 50.9 \\
\hline Belgium. ......... & 122.7 & 116.5 & $544 \cdot \mathrm{I}$ & $27 \cdot 7$ \\
\hline 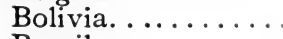 & $3 \cdot 6$ & $3 \cdot 7$ & 6.2 & 0.3 \\
\hline Brazil... & $137 \cdot 3$ & 99.4 & 540.7 & 23.9 \\
\hline Bulgaria.......... & I 8.9 & 189 & 62.4 & $5 \cdot \mathrm{r}$ \\
\hline 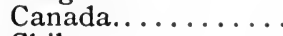 & $5^{8.1}$ & 50.8 & 271.8 & I 3.8 \\
\hline Chile.... & 38.7 & 44.0 & $107 \cdot 3$ & $4 \cdot 7$ \\
\hline China ... & 62.7 & 71.9 & $613 \cdot 1$ & 30.4 \\
\hline Colombia. ... & & & $14 \cdot 5$ & \\
\hline Costa Rica... & 2.8 & 2.8 & 14.6 & 0.6 \\
\hline Cuba....... & 18.8 & 19.5 & & \\
\hline Denmark........... & 20.3 & 208 & 66.0 & 2.2 \\
\hline Dutch East Indies. & 61.9 & 66.8 & & \\
\hline Ecuador. ......... & $5 \cdot 2$ & $4 \cdot 5$ & $5 \cdot 7$ & 0.4 \\
\hline Egypt....... & 60.1 & $5^{6} \cdot 5$ & 500.7 & $2 \mathrm{I} .7$ \\
\hline 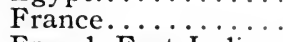 & $695 \cdot 3$ & $695 \cdot 3$ & $5^{8} 5^{6} \cdot 7$ & 227.8 \\
\hline French East Indies. & 12.0 & 12.0 & & \\
\hline Germany ......... & $495 \cdot 9$ & 553.2 & 698.8 & 23.6 \\
\hline Greece. . . . . . . . . & $14 \cdot 7$ & $14 \cdot 3$ & I 59.8 & $4 \cdot 5$ \\
\hline Guatemala... . & 2.0 & 2.2 & I 2.1 & 0.7 \\
\hline Haiti......... & $7 \cdot 3$ & $7 \cdot 3$ & 28.0 & 3.0 \\
\hline Honduras... . . . . . & I. 4 & I. 3 & 96.2 & 0.2 \\
\hline India. . . . . . . . . & $37 \mathrm{I} \cdot 5$ & 346.4 & I 102.9 & 36.2 \\
\hline Italy $\ldots \ldots \ldots \ldots \ldots$ & 375.0 & $35^{6.5}$ & 2560.6 & I 14.4 \\
\hline Japan............ & 142.9 & $142 \cdot 5$ & 261.9 & 21.7 \\
\hline Korea.......... & $5 \cdot 4$ & $5 \cdot 4$ & & \\
\hline Mexico....... & 29.2 & 27.8 & I $75 \cdot 9$ & $9 \cdot I$ \\
\hline Netherlands.... & $61 \cdot 5$ & $61 \cdot 5$ & $463 \cdot 2$ & 14.0 \\
\hline New Zealand....... & 31.4 & 30.2 & $275 \cdot 4$ & $9 \cdot 3$ \\
\hline Nicaragua. . ....... & 2.4 & 2.4 & 5.6 & 0.2 \\
\hline Norway. .......... & 27.0 & $27 \cdot 3$ & 70.4 & 2.7 \\
\hline
\end{tabular}


REVENUE, ETC.-Continued.

\begin{tabular}{|c|c|c|c|c|}
\hline & Revenue. & $\begin{array}{l}\text { Expendi- } \\
\text { ture. }\end{array}$ & Debt. & Interest. \\
\hline Paraguay......... & I I .O & I I O O & I I . 2 & 0.2 \\
\hline 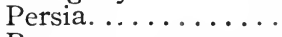 & $7 \cdot 3$ & $7 \cdot 3$ & I 6.7 & I. O \\
\hline Peru. . . . ...... & 75 & 70 & $23 \cdot 2$ & \\
\hline Philippine Islands. . & I $5 \cdot 3$ & I $4 \cdot 3$ & 60 & 0.2 \\
\hline Portugal........ & 573 & 62.2 & $8 \div 9 \cdot 9$ & $22 \cdot 7$ \\
\hline Rumania......... & $42 \mathrm{I}$ & 38.9 & 272.8 & I 6.7 \\
\hline 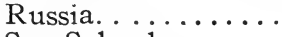 & I I 26.7 & I I 4 I. I & 3440.0 & I $52 \cdot 3$ \\
\hline $\begin{array}{l}\text { an Salvador........ } \\
\text { anto Domingo..... }\end{array}$ & $\begin{array}{l}3 \cdot 3 \\
\text { I. } 9\end{array}$ & $\begin{array}{l}3 \cdot 3 \\
\text { I. } 7\end{array}$ & $\begin{array}{r}3 \cdot 7 \\
26 \cdot 2\end{array}$ & 0.2 \\
\hline 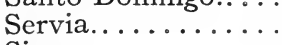 & I3.6 & I $4 \cdot I$ & 80.8 & $3 \cdot 7$ \\
\hline $\operatorname{iam} \ldots \ldots \ldots . . .$. & I 3.8 & I36 & & \\
\hline 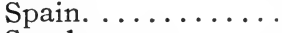 & I $97 \cdot \mathrm{I}$ & 187.8 & $206 I \cdot 4$ & 80.4 \\
\hline 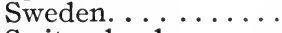 & $49 \cdot 7$ & $49 \cdot 6$ & 92.8 & $3 \cdot 2$ \\
\hline witzerland....... & 20.7 & 20.6 & I $7 \cdot 4$ & 0.8 \\
\hline Turkey ........... & $8 I \cdot 5$ & $8 \mathrm{I} \cdot \mathrm{I}$ & $723 \cdot I$ & $28 \cdot 4$ \\
\hline United Kingdom. . . & $737 \cdot 5$ & $897 \cdot 8$ & $3885 \cdot 2$ & I 38.4 \\
\hline United States...... & 694.6 & $640 \cdot 3$ & 925.0 & 28.6 \\
\hline Uruguay. $\ldots \ldots .$. & I6. 7 & I 5.0 & I $27 \cdot 4$ & $6 \cdot 7$ \\
\hline Venezuela....... & & 5.0 & $40 \cdot 3$ & \\
\hline
\end{tabular}

\section{RAILWAY MILEAGE.}

\begin{tabular}{|c|c|c|c|}
\hline & $\begin{array}{l}\text { Miles. } \\
\text { I,920 }\end{array}$ & Chin & $\begin{array}{r}\text { Miles. } \\
2,870\end{array}$ \\
\hline . & II, 460 & Colombia... & 4 I I \\
\hline & I 4,789 & Costa Rica.. & 340 \\
\hline Hungary . & 24,0 & & $\mathbf{I}, 479$ \\
\hline$\ldots \ldots$ & 2,843 & $\operatorname{ark} \ldots \ldots$ & $\mathrm{I}, 9 \mathrm{I} 2$ \\
\hline & 700 & East Indies & 1,392 \\
\hline & 10,408 & $\cdots$ & I 25 \\
\hline nies, & & $\mathrm{Eg}$ & 3,400 \\
\hline & 2,394 & & 27,870 \\
\hline uth Africa & 7,139 & Colonies, & \\
\hline & 302 & & I, 2 I 6 \\
\hline & $\begin{array}{r}1,020 \\
19,078\end{array}$ & $\begin{array}{l}\text { French East Indies } \\
\text { German Colonies.. }\end{array}$ & 320 \\
\hline & 2,800 & Germany........ & 34,3 I 4 \\
\hline
\end{tabular}


RAILWAY MILEAGE-Continued.

Greece. .........

Guatemala......

Haiti. .

Honduras.........

India. ...........

Italy............

Japan. .........

Korea.

Mexico..........

Netherlands.

New Zealand.....

Nicaragua.

Norway.

Panama

Paraguay........

Persia.

Peru.

Philippine Islands Portugal.
700 400

43

57

26,956 9,960 $4,65 \mathrm{I}$ 326 12,070 I,809 2,525

2 I0 I, 48 ०

47

156

I , I 46

I 20 I,486
Rumania

Russia.

Salvador.

Santo Domingo...

Servia..........

Siam.

Spain.

StraitsSettlements

Sweden.........

Switzerland.

Tunis.

Turkey.

United Kingdom. 22,435

United States.... 207,784

Uruguay...... I, 210

Venezuela.

529

World. . . . . 5 535,845

\section{TONNAGE OF MERCHANT SHIPPING.}

IN THOUSANDS OF TONS.

Australia...

Austria-Hungary.

Belgium

Canada.

China

Denmark

France.

Germany.

India.

Italy.

Japan

Netherlands.

New Zealand.

Norway.

Sail.

Russia.

Spain.

Sweden.

United Kingdom.

United States.
$14 \mathrm{I}$

22

I

Steam. Total. 208 282

349 304 I I O 653

2 I

I 57

564

587

20

575

335

75

43

936

556

95

299

r 991

2603
IO9

20

259

547

I 506

48

425

583

307

62

$53 \mathrm{I}$

418

679

$34 \mathrm{I}$

7618

292 I

\section{$4 I$}

416

2093

68

I 000

9 I 8

382

I0 5

r 467

974

774

640 9609

5524
I I I I 


\section{PRODUCTION OF ALUMINUM.}

France

Metric Tons.

Switzerland.

United Kingdom. . . . . . . . . . . . . .

United States.

I, 700

2,500

650

3,402

World.

$8,25^{2}$

\section{PRODUCTION OF ANTIMONY.}

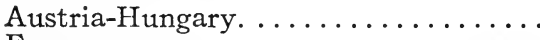

France

Germany.

Short Tons.

Italy

Japan.

Servia..

United States.

833

3,029

3,537

$\mathrm{I}, 2 \mathrm{O} 2$

647

379

570

\section{PRODUCTION OF ARSENIC.}

Canada.

Metric Tons.

66

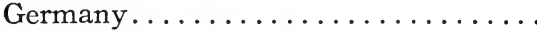

Portugal

Spain.

United Kingdom.

United States.

2,768

736

$\mathrm{I}, \mathrm{O} 88$

902

36

\section{PRODUCTION OF GRAPHITE.}

Austria-Hungary $\ldots \ldots \ldots \ldots \ldots \ldots \ldots$

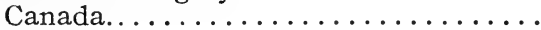

Ceylon.

Metric Tons.

Germany

29,992

2,005

India. .

22,707

4,435

2,530

Italy.

I0,3 13

Mexico

762

United States.

2,533

World.

75,277 
STATISTICAL ABSTRACT OF THE WORLD. 23

\section{PRODUCTION OF SULPHUR.}

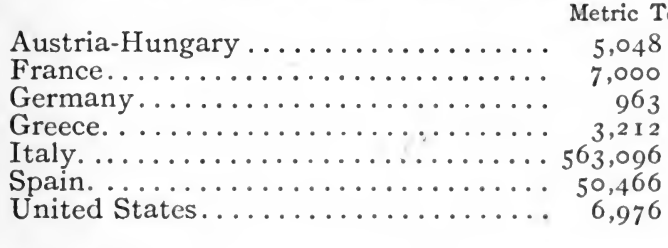

World. ..............6.6.6.

\section{PRODUCTION OF BORAX.}

Chile................... II 547

Germany................. $\quad$ I 84

India. ................... I62

Italy.................... $2,55^{8}$

Peru.................... 7,080

Turkey................... I 2,000

United States.............. 16,227

\section{PRODUCTION OF PETROLEUM.}

IN Millions of BarRels.

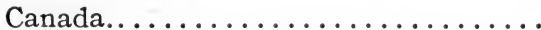

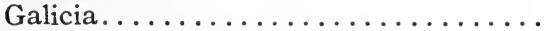

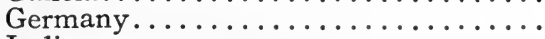

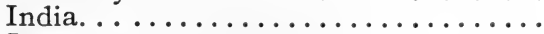

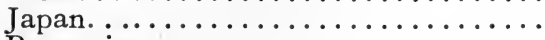

Rumania.

Russia.

Sumatra, Java, and Borneo.......... United States. 


\section{PRODUCTION OF ASPHALTUM.}

In Thousands of Tons.

Austria-Hungary.

France.

Germany. .

Italy.

Russia.

Spain.

Trinidad.

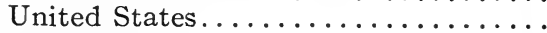

Venezuela.

\section{PRODUCTION OF MANGANESE ORE.}

In Thousands of Long Tons. Australia

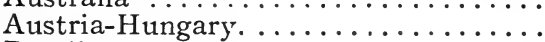

Brazil.

Chile.

Cuba.

France.

Germany.

Greece.

India.

Italy

Russia.

Spain.

Sweden.

\section{PRODUCTION OF TIN.}

Australia.

Long Tons.

Banka and Billiton 5,082 Bolivia.

Malay States................ 5 58,657

World. ................... 73,499 


\section{PRODUCTION OF ZINC.}

In Thousands of Long Tons.

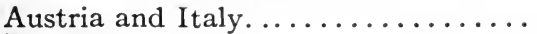

Belgium.

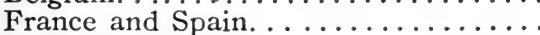

Netherlands.

Poland.

Rhine District.

Silesia.

United Kingdom.

9
138

48

I3

I0

64

I 23

45

United States.............. I67

World................... 6 r 9

\section{PRODUCTION OF COAL.}

In Thousands of Short Tons. Australia . . Austria-Hungary

Belgium.

Canada.

26,224

7,644

Cape Colony.

France.

38,467

Germany 186,732

India.

$8,33 \circ$

Italy.

382

Japan.

I 0,691

Mexico.

Natal.

799

Netherlands.

440

New Zealand...................

Russia.

1,590

Spain.

19,285

Sweden

$2,85^{2}$

Transvaal

353

United Kingdom.

2,529

United States.

260,3 I 3 $35^{2,310}$

World. 978,088 


\section{PRODUCTION OF COPPER.}

In Thousands of Long Tons.
Australia. . . . $\ldots \ldots \ldots \ldots \ldots \ldots \ldots \ldots$

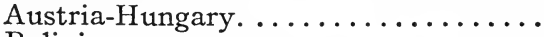

Bolivia.

Canada and Newfoundland. .........

Cape Colony. ..................

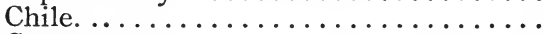

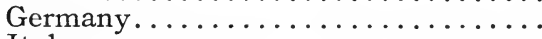

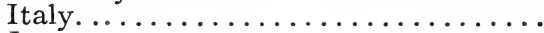

Japan.

Mexico.

Norway.

Peru.

Russia.

Spain and Portugal.

United States.

World.

\section{PRODUCTION OF IRON ORE.}

Algeria.

In Thousands of Long Tons.

Australia

Austria-Hungary.

Belgium.

Canada.

Cuba.

France.

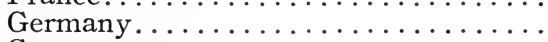

Greece.

India.

$2 \mathrm{I}, 23 \mathrm{I}$

Italy

Japan.

375

Russia......................

Spain.

70

Sweden.

3,648

8,304

United Kingdom.

3,678

United States.

World................... ror, 785 


\section{PRODUCTION OF SALT.}

IN THOUSANDS OF SHORT TONS.

Austria-Hungary.

$$
\begin{array}{r}
576 \\
62 \\
x, 096 \\
1,867 \\
1,002 \\
505 \\
762 \\
1,913 \\
470 \\
2,113 \\
2,656
\end{array}
$$

France.

Germany

India.

Italy.

Russia.

United Kingdom

United States

World................... r 3,023

\section{PRODUCTION OF QUICKSILVER.}

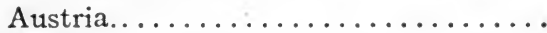

Metric Tons.

Italy 536

Russia

355

Spain.

393

United States.

1,020

I, $\mathrm{I} 88$

World. .

$3,49^{2}$

\section{MANUFACTURE OF PIG IRON AND STEEL.}

IN THOUSANDS OF LONG TONS.

Pig Iron. Steel.

Austria-Hungary............. I, 428

Belgium................. 1, 217

Canada..................... ${ }_{26} 65$

France................ 2,84 I

Germany................ 10,086

Italy.

75

Russia. ................ 2,454

Spain.

$3 \circ 3$

Sweden.................... 507

United Kingdom. ............ 8,935

United States.............. r 8,009

I , I 93

969

I 82

I, 885

8,802

I 87

2,375

200

3 I 9

$5, \mathrm{I} 34$

I 4,535

World. . . .............. 46,368 


\section{PRODUCTION OF GOLD AND SILVER.}

IN ThOUSANDS OF DOLLARS.

Commercial Value.

Gold.

Africa.

67,998

Silver.

Argentina. ................ 30

Australasia. ................ 89,2 10

Austria-Hungary. ........... 2,245

Bolivia............ I

Brazil.

2,274

British East Indies. . . . . . . .

British Guiana............... I,6 I I

Canada................. I 8,835

I 85

Central America. ............ I, 875

Chile.

667

China................ 7,325

Colombia............. 2,724

Dutch East Indies........... 502

Dutch Guiana............... $\quad 376$

Ecuador.................. $\quad 274$

Finland. ................ 2

France.

French Guiana. . . . . . . . . . .

Germany..................

Greece.

2,102

$7 \mathrm{I}$

India.

I I , 429

Italy.

Japan.

2,003

Korea.

3,000

Mexico................. 10,678

Norway. .................

Peru.

Portugal............... I

Russia. . ................. $24,63^{2}$

Spain. ............... 5

Sweden.................. 34

Turkey................. 2 I

United Kingdom. ............ 77

United States............ 73,592

Uruguay. . . .............. ${ }^{51}$

Venezuela. ................ 85

4,843

$\mathrm{I}, 7 \circ \mathrm{I}$

I , I 43

I, 402

6 10

66

5
404

$3, \mathrm{I} 44$

735

435

293

38,070

I07

943

82

2,209

I8

248

79

29,322

World. . . . . . . . . . . . . 325,527 


\section{CONSUMPTION OF COPPER.}

In Thousands of Metric Tons.

Austria-Hungary............. I9

Belgium................... 6

France..................... 49

Germany.................... I I0

Italy .................... I0

Netherlands................. 2

Russia.................... I8

United Kingdom. ................ 108

United States................. 230

Other countries................ 20

World. . . . . . . . . . . . . $57^{2}$

\section{PRODUCTION OF POTATOES.}

In Millions of Bushels.

Australia.................. I5

Austria-Hungary. ................ 659

Belgium................

Canada......................... 30

Denmark. .................. 2 I

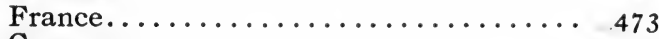

Germany.................. I 7 I I

Italy..................... ${ }_{28}$

Japan................... ro

Netherlands...................... 78

New Zealand.................. 7

Norway..................... 22

Rumania................... 5

Russia....................... I08 I

Sweden................... 55

United Kingdom. .............. 196

United States................. 273 
30 STATISTICAL ABSTRACT OF THE WORLD.

\section{GRAIN CROPS OF THE PRINCIPAL COUNTRIES}

In Millions of Bushels.

\begin{tabular}{|c|c|c|c|c|c|}
\hline & Corn. & Wheat. & Oats. & Barley. & Rye. \\
\hline Algeria. . . & & 30 & 5 & 46 & \\
\hline Argentina. & 84 & 122 & & & \\
\hline Australia........ & & 82 & 8 & $I$ & \\
\hline Austria-Hungary. . . . . & I33 & 224 & 222 & I45 & I32 \\
\hline Belgium. ............ & & I 3 & 43 & 5 & 22 \\
\hline Bulgaria.. & I 8 & 34 & 7 & Io & 8 \\
\hline Canada..... & 2 I & 80 & I 70 & 39 & 4 \\
\hline Cape Colony. ........ & 2 & 4 & 2 & $\mathrm{I}$ & \\
\hline Chile............ & 9 & I 3 & & & \\
\hline Denmark............ & & 4 & 36 & 23 & I8 \\
\hline Egypt............. & 30 & 12 & & & \\
\hline France............ & 23 & 365 & 315 & 47 & 60 \\
\hline$y \ldots \ldots \ldots$ & & I35 & $54^{2}$ & I 53 & 390 \\
\hline $\begin{array}{l}\text { Greece. . . . . . . . } \\
\text { India. . . . }\end{array}$ & & $\begin{array}{r}7 \\
302\end{array}$ & & & \\
\hline Italy............... & $7 \mathrm{I}$ & I62 & I6 & 8 & 4 \\
\hline$\ldots \ldots \ldots \ldots$ & & 23 & & 82 & \\
\hline 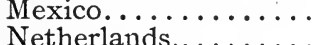 & 78 & & & 9 & \\
\hline 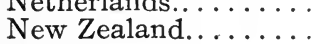 & & 5 & $\begin{array}{l}19 \\
22\end{array}$ & $\begin{array}{l}4 \\
\mathrm{I}\end{array}$ & 13 \\
\hline Persia........... & & 23 & & & \\
\hline Portugal.. & I6 & 8 & & & \\
\hline Rumania. & 68 & 74 & $3^{I}$ & 30 & 7 \\
\hline Russi & 49 & $45^{I}$ & 800 & 357 & 9I3 \\
\hline ....... & 22 & I I & & & \\
\hline Spain. & 22 & 102 & II & 60 & 20 \\
\hline Sweden...... & & 4 & 63 & I 5 & 24 \\
\hline $\begin{array}{l}\text { Switzerland. . } \\
\text { Turkey..... }\end{array}$ & & 4 & & & \\
\hline 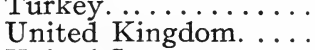 & & $\begin{array}{l}41 \\
49^{\circ}\end{array}$ & I 87 & 67 & 2 \\
\hline United States....... & 2666 & 659 & 943 & I 20 & 26 \\
\hline World. & 3182 & $306 \mathrm{I}$ & 3284 & I 242 & I 646 \\
\hline
\end{tabular}




\section{PRODUCTION OF TOBACCO.}

In Millions of Pounds.

Algeria................. I3

Australia.................. I

Austria-Hungary................ I 50

Belgium................... I0

Brazil...................... 55

Bulgaria.................. 19

Canada....................... ro

Cape of Good Hope.............. 5

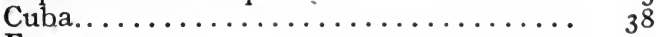

France.................... 57

Germany................... 73

Greece. .................. $\mathrm{r}_{4}$

India. ................. r 4 I

Italy. .............. I 3

Japan...................... $9^{6}$

Java and Sumatra............... r Io

Mexico..................... 20

Natal. ............... 4

Netherlands................. 2

Philippine Islands. . . . . . . . . . 37

Porto Rico................... 5

Rumania.................. I0

Russia................... I 74

Sweden.................... 2

Turkey.................... 66

United States.................... 868

World.......................2205 


\section{PRODUCTION OF CANE=SUGAR.}

In Thousands of Long Tons.

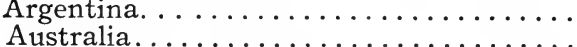

Brazil.

I 27

I 57

British Guiana. ............... roo

British West Indies............... I 2 I

Central America................. 23

Cuba.................... I 75

Dutch Guiana................. r 3

Egypt..................... 90

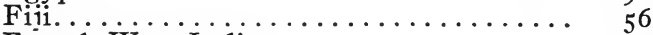

French West Indies.................... 6 I

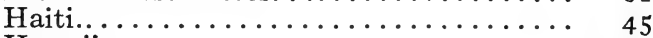

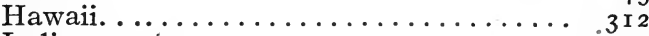

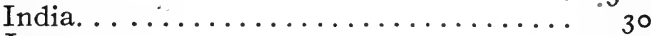

Java...................... I

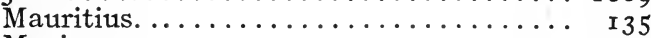

Mexico................... I I 5

Peru....................... I40

Philippine Islands. ............... I 80

Porto Rico.................... I 55

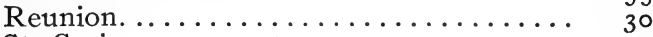

St. Croix. ................ I I

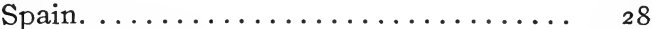

United States................. 330

Venezuela.................... 3

West Indies (other)............... I3

World........................ 4588

PRODUCTION OF BEET-SUGAR.

Austria................... 893

Belgium.................. I73

France...................... 625

Germany................... ${ }_{575}$

Netherlands.................. I 35

Russia. ..................... 940

United States................. 2 ro

World...................... 489 


\section{CONSUMPTION OF SUGAR PER CAPITA.}

Lbs.

Austria-Hungary................ 17.6

Belgium.................... 23.3

Bulgaria................... 6.7

Denmark. . ................. 54.8

France....................... 37.0

Germany................... 33.9

Greece. ................... 7.2

Italy..................

Netherlands................. 32.5

Portugal. .................. 14.7

Rumania................... 7.8

Russia. ................... 14.0

Servia..................... 5.3

Spain. .................... ro.6

Sweden and Norway............... 38.2

Switzerland. .................. 60.3

Turkey.................... 8.0

United Kingdom. .............. 91.6

United States................. 65.2

\section{IMPORTATIONS OF SUGAR TO UNITED STATES}

IN Millions of Pounds.

Austria-Hungary ............... 4

Brazil. ................... I4

British Guiana. ................... 73

British West Indies............... 66

Canada....................... 4

China...................... 5

Cuba........................2820

Danish West Indies.............. 2 I

Dutch East Indies.............. 440

Dutch Guiana.................. 7

Egypt....................... 22

Germany................... 5

Hawaii. ................ 504

Mexico.................... I

Peru....................... 49

Philippine Islands. ............. 62

Porto Rico. . . . . . . . . . . . . . . . 73

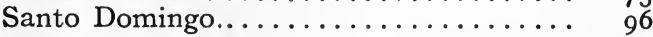

World....................... 3700 


\section{PRODUCTION OF COFFEE. SOURCES OF SUPPLY.}

Exports in Millions of Pounds.

Brazil. ...................... I600

British East Indies. . . . . . . . . . 68

British West Indies. . . . . . . . . I I

Central America. ................. I6 5

Colombia, Venezuela, Ecuador, Peru, and

Chile.................... 200

Cuba and Porto Rico............. I8

Dutch East Indies. .............. I 35

Haiti and Santo Domingo........... 7 I

Mexico..................... 35

\section{CONSUMPTION OF COFFEE.}

In Millions of Pounds.

Austria-Hungary .............. r... 109

Belgium.................... I 25

Canada..................... 6

France................... I 68

Germany.................. 396

Netherlands. ................... 29

United Kingdom. .............. 29

United States............... 96I

\section{PRODUCTION OF TEA. SOURCES OF SUPPLY.}

Exports In Millions of Pounds.

Ceylon. ................. 149

China.................... 193

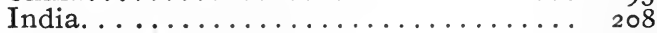

Japan. ...................... 68

Java...................... 24 


\section{CONSUMPTION OF TEA.}

In Millions of Pounds.

Australia................... 29

Canada...................... 24

France..................... 2

Germany.................. 7

Netherlands. . . . . . . . . . . . . 9

Russia................... I 2 I

United Kingdom. .............. 257

United States................. I I0

\section{COCOA.}

Production in Millions of Pounds.

Brazil.

Colombia...............

Ecuador................... 47

Portuguese Africa. ............... 45

Santo Domingo................ I $_{5}$

Trinidad..................... 29

Venezuela................... $\quad 25$

Consumption in Millions of Pounds.

France................

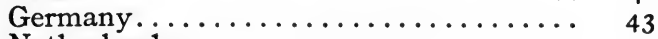

Netherlands. . . . . . . . . . . . . 33

Spain. $\ldots \ldots \ldots \ldots \ldots \ldots \ldots \ldots \ldots \ldots \ldots{ }_{13}$

United Kingdom. .................. 35

United States................. $6_{3}$

\section{PRODUCTION OF FLAX.}

\begin{tabular}{|c|c|c|}
\hline & $\begin{array}{l}\text { Seed, } \\
\text { Thousands of } \\
\text { Bushels. }\end{array}$ & $\begin{array}{l}\text { Fiber, } \\
\text { Thousands of } \\
\text { Pounds. }\end{array}$ \\
\hline Argentina. & 30,076 & - \\
\hline Austria-Hungary. & $\mathrm{r}, 3 \mathrm{I} 4$ & I 26,848 \\
\hline elgium. . . . . . . . . & 272 & 24,790 \\
\hline Bulgaria............... & 23 & 2,116 \\
\hline lada.................... & 582 & \\
\hline
\end{tabular}




\section{PRODUCTION OF FLAX-Continued.} Thousands of

France............. 500

India. ................ I9, ${ }^{2} 6_{3}$

Ireland.

Italy.

Mexico.

Netherlands $\ldots \ldots \ldots \ldots$

Rumania.............

Russia.............

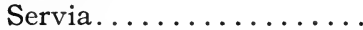

Sweden.

United States.
Seed, Bushels.

Fiber, Thousands of Pounds.

47,000

I 9,327 $4 \mathrm{I}, 9$ I 7

150
362
2,064
17,997
11
60

27,301

\section{SILK PRODUCTION.}

In Millions of Pounds.

Austria-Hungary.................. 0.6 China..................... r $3 . \mathbf{I}$

Europe, Southeastern. . . . . . . . . . 2.7 France..................... r.o

India. ....................... 0.5

Italy.................

Japan...................... ro.2

Levant. .................... 2.8

Spain. .................. 0.2

\section{VALUE OF SILK MANUFACTURES.}

In Millions of Dollars.

Austria................... I7

France..................... I 22

Germany................... 73

Italy....................... I3

Russia.................. 2 I

Spain and Portugal............. 4

Switzerland. ................... $3^{8}$

United Kingdom. ............. I5

United States............... $9^{2}$

World.................... 395 


\section{COTTON PRODUCTION.}

In Thousands of Bales of 500 Lbs.

Brazil.

Chile.

China

Egypt

I, 200

Greece.

I, 164

India.

2,688

Japan. . . . . . . . . . . . . . .

Java.

5

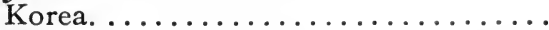

Malta.

400

Mexico

Persia.

I 24

Peru.

Russia.

Turkey.

354

United Sta

80

$10,63 \mathrm{I}$

World.

\section{COTTON MANUFACTURES.}

In Thousands of Bales.

Austria-Hungary. . . . . . . . . .

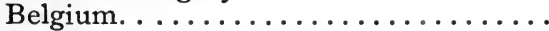

$54 \mathrm{I}$

Canada.

France.

I I I

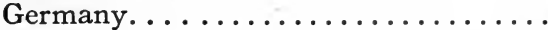

832

Greece.

I, 259

India.

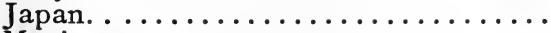


COTTON MANUFACTURES-Continued.

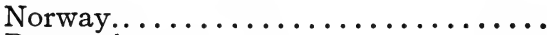

Portugal.

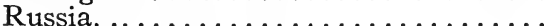

Spain.

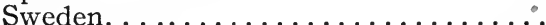

Switzerland.

United Kingdom. ................ United States.

\section{COTTON SPINDLES.}

In Millions.

Austria-Hungary...............

Belgium.

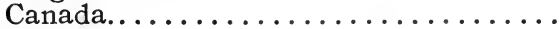

China

0.6

France.

$5 \cdot 0$

Germany.................. 7.2

Greece................... I.

India.................... 4.9

Italy................... 2.I

Japan................... I. 2

Mexico...................... o. 5

Netherlands.................... ० 3

Norway................... o.r

Portugal. .................. 0.2

Russia..................... $7 \circ$

Spain..................... 2.6

Sweden.................... 0.4

Switzerland. ............. I.7

United Kingdom. .............. 454

United States................ I8.6

World..................... 103.4 


\section{WOOL PRODUCTION.}

\section{In Millions of Pounds.}

Africa, not specified............ I

Algeria and Tunis................ 30

Argentina. ............................ 370

Asia, Central.................. ${ }_{46} 6$

Asia, not specified............... I 5

Australasia. ................... 480

Austria-Hungary................ 64

Brazil. ................... 2

Canada and Newfoundland......... 12

Central America and West Indies.........
Chile........................

China........................... 35

Egypt.................... 3

Europe, not specified............. I4

France.................... 9 r

Germany......................... 50

India........................ 85

Italy..................

Mexico...................... ro

Portugal. . . .............. I

Russia..................... 42 I

South Africa................... I00

South America, not specified........... 20

Spain. .................. ro3

Sweden and Norway.............. 8

Turkey and the Balkan Peninsula....... ror

United Kingdom. ............... r 33

United States.................. 292

Uruguay. . . . . ................. ${ }_{96}^{6}$

Venezuela. .................. 15 
40 STATISTICAL ABSTRACT OF THE WORLD.

\section{LIVE STOCK.}

In MiLlions.

\begin{tabular}{|c|c|c|c|c|c|}
\hline & Horses. & Cattle. & Sheep. & Hogs. & Goats. \\
\hline 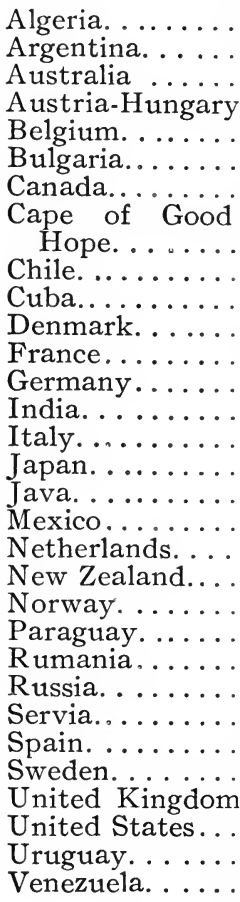 & 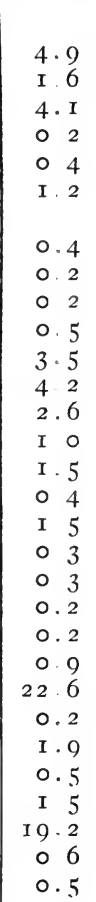 & 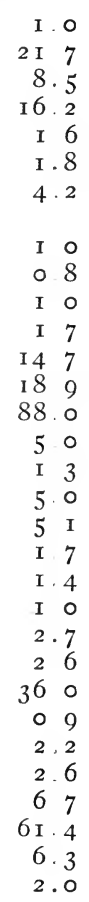 & $\begin{array}{rr}7 & 6 \\
74 & 4 \\
72 & 2 \\
10 & 7 \\
6 & 9 \\
2 & 6 \\
\text { I } 2.6 \\
1 & 3 \\
& \\
\text { 1. } & \\
\text { I } 9.7 \\
9 & 7 \\
\text { I } 7 & 8 \\
6 & 9\end{array}$ & $\begin{array}{r}0.6 \\
0.7 \\
0.2 \\
0.2 \\
\text { I. } \\
\text { I2.0 } \\
0.9 \\
\text { I.9 } \\
0.8 \\
2.7 \\
48.7\end{array}$ & $\begin{array}{l}4.2 \\
0.2 \\
0.2 \\
0.2\end{array}$ \\
\hline
\end{tabular}




\section{FISHERIES.}

In Millions of Dollars.

Belgium................... I

Canada........................ 46

Denmark. ................... 3

Italy..................... 3

Japan.................... I3

Norway. ................... 8

Portugal. . . . . . . . . .

Spain................... 8

United Kingdom. .............. ${ }_{46} 6$

United States............... 49

World................... I 8 I

\section{PRODUCTION OF WINE.}

\section{In Millions of Gallons.}

Algeria............... I 58

Argentina. . . . . . . . . . . . ${ }_{26}$

Australia.................... 7

Austria.................. I 24

Brazil..................... 7

Bulgaria.................... 53

Chile....................... 6 т

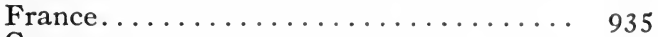

Germany.................... $5_{50}$

Greece. . . . . . . . . . . . . . . . . 34

Hungary. . . . . . . . . . . . . . 55

Italy...................... 898

Portugal. . . . . . . . . . . . . . $9^{2}$

Rumania..................... 34

Russia. .................... 74

Servia.................... I 2

Spain. ..................... 396

Switzerland. ................ ${ }_{\text {I } 6}$

Tunis. ................

Turkey................... 55

United States................. 45

World...................... 31 62 


\section{CONSUMPTION OF LIQUORS.}

In Millions of Gallons.

\begin{tabular}{|c|c|c|c|}
\hline & Beer, etc. & Wines. & Alcohol. \\
\hline 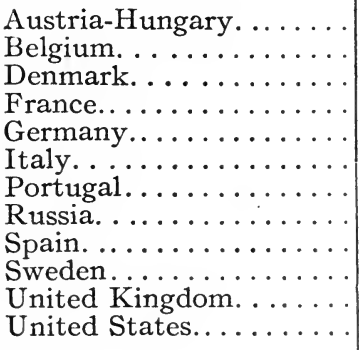 & $\begin{array}{r}546 \\
395 \\
63 \\
289 \\
\times 783 \\
7 \\
- \\
\text { I } 52 \\
20 \\
44 \\
\text { I } 500 \\
\text { I } 494\end{array}$ & $\begin{array}{r}\text { I } 80 \\
- \\
\text { I } 343 \\
\text { I } 14 \\
\text { I046 } \\
\text { I } 20 \\
25 \\
400 \\
\text { I } \\
\text { I } 7 \\
43\end{array}$ & $\begin{array}{r}\text { I } 20 \\
\text { I0 } \\
4 \\
97 \\
\text { I } 24 \\
\text { I I } \\
\\
\text { I } 73 \\
\text { I I } \\
58 \\
\text { I } 21\end{array}$ \\
\hline
\end{tabular}

\section{WOODED AREAS.}

Per Cent of Total Area.

Austria...................... 32

Belgium. .................. I7

Bosnia and Herzegovina. ........... 53

Bulgaria...................... 45

Denmark. ................... 6

France................... I 8

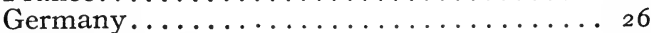

Greece. ..................... I6

Hungary. .................... 28

Italy. .................... I4

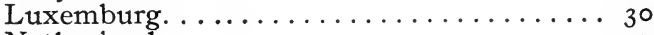

Netherlands................... 7

Norway. .................. 2 I

Philippine Islands. . . . . . . . .

Portugal. .................. 3

Rumania.................... I7

Russia in Europe.................. 40 


\section{WOODED AREAS-Continued.}

Per Cent of Total Area.

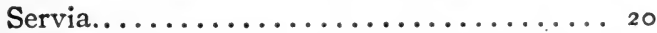

Spain.................... r 7

Sweden..................... 44

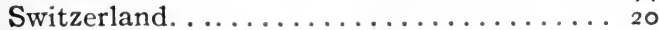

Turkey in Europe. ............... 8

United Kingdom. . ............... 4

United States................... 37

\section{CULTIVATED AREAS.}

Argentina..................

Australia..................... r

Austria....................... 35

Belgium................... 57

Cuba......................... 5

Denmark........................ $7^{5}$

Egypt..................... 4

England and Wales................. 74

France....................... 48

Germany................... $4^{2}$

Greece. .................... 22

Hawaii. .................... 7

India....................... 20

Ireland................... 22

Italy...................... 7 I

Netherlands ................ 62

New Zealand.................... 20

Norway................... I

Philippine Islands. . . . . . . . . . . 5

Porto Rico.................. $2 \mathbf{I}$

Portugal...................... 25

Rumania.................... 43

Russia in Europe................. 24

Servia........................... 30

Spain...................... 25

Sweden..................... 8

United States................. 2 I 
44 SIATISTICAL ABSTRACT OF THE WORLD.

\section{FOREIGN COMMERCE.}

\section{In Millions of Dollars.}

Algeria............... Argentina. .............

Austria-Hungary......... Belgium.

Bolivia.

Brazil.

British South Africa.....

British West Indies.

Bulgaria.............

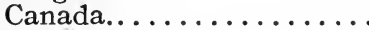

Chile.

China

Colombia.............

Costa Rica.............

Cuba................

Denmark.

Dutch East Indies. .......

Ecuador..............

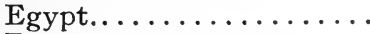

France.

French East Indies. ......

Germany.............

Greece. ..............

Guatemala............

Haiti................

Honduras. . . . . . . . . . .

India. . . ............

Italy................

Japan. ...............

Korea...............

Mexico...............

Netherlands............

New Zealand............

Nicaragua. ............

Norway. .............

Panama
Imports. Exports. Total.

\begin{tabular}{|c|c|c|}
\hline 64 & $6 I$ & I 25 \\
\hline 99 & I 73 & 272 \\
\hline 349 & 388 & 737 \\
\hline 459 & 372 & 831 \\
\hline 5 & I I & 16 \\
\hline I I 3 & I77 & 290 \\
\hline 243 & I03 & 346 \\
\hline $3^{2}$ & 29 & 61 \\
\hline$I_{4}$ & 20 & 34 \\
\hline 225 & I96 & $42 \mathrm{I}$ \\
\hline 48 & 68 & I 6 \\
\hline I 98 & I35 & 333 \\
\hline 8 & I 7 & 25 \\
\hline 4. & 6 & IO \\
\hline 59 & 78 & I 37 \\
\hline II 7 & 86 & 203 \\
\hline 87 & 99 & 186 \\
\hline 7 & 9 & I6 \\
\hline 73 & 87 & I 60 \\
\hline 848 & 821 & I 669 \\
\hline 42 & 4I & 83 \\
\hline$I, 340$ & I, I I I 3 & 2,453 \\
\hline 26 & I 5 & $4 I$ \\
\hline 3 & 7 & IO \\
\hline 5 & I 3 & I8 \\
\hline 2 & 2 & 4 \\
\hline $25^{6}$ & 408 & 664 \\
\hline 343 & 284 & 627 \\
\hline I 40 & I 34 & 274 \\
\hline 7 & 4 & II \\
\hline 75 & 88 & I63 \\
\hline 867 & 733 & 1,600 \\
\hline 55 & 66 & I $2 \mathrm{I}$ \\
\hline 2 & 3 & 5 \\
\hline 78 & 46 & I 24 \\
\hline 3 & I & 4 \\
\hline
\end{tabular}


FOREIGN COMMERCE-Continued.

\begin{tabular}{|c|c|c|c|}
\hline Paraguay............... & 2 & 4 & 6 \\
\hline Persia................ & 24 & I 3 & 37 \\
\hline Peru................ & $2 \mathrm{I}$ & I 8 & 39 \\
\hline Philippine Islands. . . . . . . & 33 & 33 & 66 \\
\hline Portugal................ & 60 & $3^{I}$ & $9 \mathrm{I}$ \\
\hline 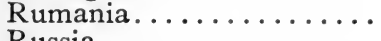 & 55 & 72 & 127 \\
\hline Russia. ............... & $35^{\mathrm{I}}$ & 431 & 782 \\
\hline Salvador. . . . . . . . . . . & 3 & 4 & \\
\hline Santo Domingo........... & 3 & 5 & \\
\hline $\begin{array}{l}\text { Servia................. } \\
\text { Siam. }\end{array}$ & 9 & 14 & 23 \\
\hline$\ldots \ldots \ldots \ldots \ldots \ldots$ & I 6 & $2 \mathrm{I}$ & 37 \\
\hline Spain. ............. & I 75 & I6 $\mathrm{x}$ & 336 \\
\hline Straits Settlements. . . . . . & I 46 & 126 & 272 \\
\hline 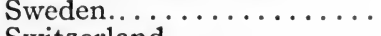 & I 35 & 105 & 240 \\
\hline Switzerland. . ........... & 218 & 169 & $3^{87}$ \\
\hline$\ldots \ldots \ldots \ldots \ldots$ & I 2 & 8 & 20 \\
\hline Turkey....................... & I 17 & 59 & I 76 \\
\hline United Kingdom. . . . . . . & $2,57 \mathrm{I}$ & $\mathbf{I}, 379$ & $3,95^{\circ}$ \\
\hline United States........... & 1,227 & $\mathrm{I}, 744$ & $2,97 \mathrm{I}$ \\
\hline$\cdots \ldots \ldots \ldots \ldots$ & 25 & 34 & 59 \\
\hline Venezuela. ............ & 9 & 15 & 24 \\
\hline
\end{tabular}

Imports. Exports. Total.

Paraguay..............

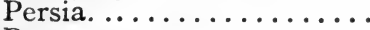

Peru..................

Portugal Islands. . . . . .

Rumania

60

Russia.

35

Salvador.

6

37

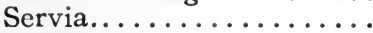

Siam.

Spain

Sweden

Switzerland.

\section{IMPORTS AND EXPORTS BY COUNTRIES.}

(In Millions of Dollars.)

Algeria.

Imports. Exports

Belgium.................

Brazil. ................. I

France.................. $5^{2}$

Morocco................. ${ }_{2}^{2}$

Spain. .............. I

Tunis.................. I

United Kingdom........... I
2 48

2

I




\section{IMPORTS AND EXPORTS BY COUNTRIES-Conid.}

(In Millions of Dollars.)

Argentina.

Belgium. .............. 5

Brazil. .................. 4

France.................... 9

Germany......... I

Italy................ I2

Paraguay....... I

Spain................ $33_{2}$

United Kingdom. ............ $3^{6} \quad 34$

United States.............. I3 I0

Australia.

Belgium................ 2

Canada.................... 2

Ceylon................ 2

France................. 2

Germany................. I3

Hongkong.......... I

India.................. 4

Japan................... 2

New Zealand............... I 3

South Africa.............. $\quad 25$

United Kingdom. ............. Ir6 98

United States.............. 24 II

Austria-Hungary.

Argentina................ 3

Belgium............... 7

Brazil................... 8

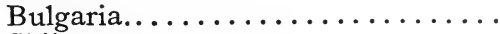

Chile................... 2

China............... I

Cuba................ I

Dutch East Indies........... 4

Egypt................. 5

France................. I 2

Germany................. I32 $20 \mathrm{I}$ 


\title{
IMPORTS AND EXPORTS BY COUNTRIES-Contd.
}

\author{
(In Millions of Dollars.)
}

Austria-Hungary-Continued.

Imports. Exports.

Greece. ................. 4

India.................... I 8

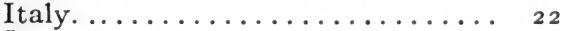

Japan................ I

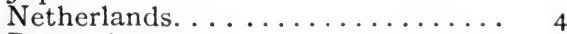

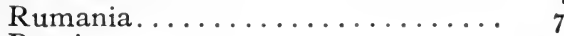

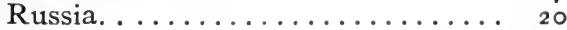

Servia................... I I

Spain.................. I

3

$8 \quad 9$

230

I I

6

I 3

Switzerland............... ro

Turkey.................... 9

United Kingdom. ............ 29

United States............... 28

\section{BELGiUM.}

Algeria................ I

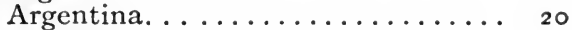

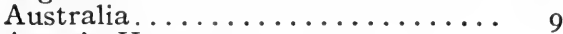

Austria-Hungary.............. $2_{2}$

Brazil.

Bulgaria.............................

Canada..................... ${ }_{2}$

Chile......................... 6

China.................... ${ }_{2}$

Denmark.

24

Egypt

- 2

France

74

Germany..................... 64

Greece.

India. . . .....................

Italy.

I 6

Japan. 


\section{IMPORTS AND EXPORTS BY COUNTRIES-Contd.}

(In Millions of Dollars.)

BELGIUM-Continued.

Imports. Exports.

Norway. ................ 4

Portugal...................

Rumania................ 28

Russia................ 28

Spain. ................. 6

Sweden.................. 9

Switzerland............. I

Turkey................. 4

United Kingdom............ 55

United States.............. 53

Bulgaria.

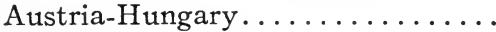

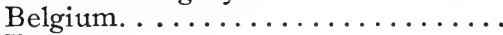

France.

Germany................ 2

Turkey.................. 2

United Kingdom.
2

2

6

8

3

5

3

69

I7

\section{Canada.}

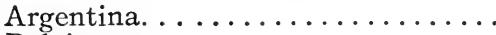

Belgium.

British Africa.

British Australasia.............

British East Indies. ........... 2

British West Indies........... 2

France................. 7

Germany............... I 2

Japan. ................. I

Netherlands. . . . . . . . . . . I

Newfoundland. ............. r

United Kingdom. ............ 59

Unitẹ Stattes..., , , , . . . . . I29

2

4

I

2

4

5

2

2

7

2

I




\section{IMPORTS AND EXPORTS BY COUNTRIES-Contd.}

(In Millions of Dollars.)

Cape Colony.

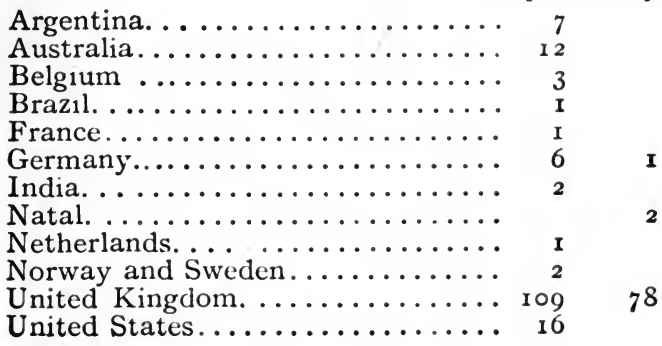

Imports. Exports.

Ceylon.

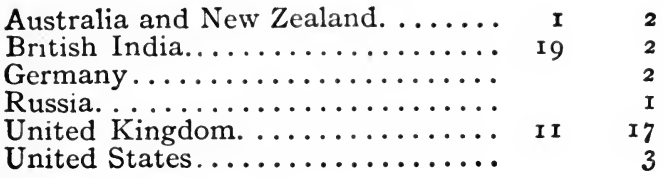

Chile.

Argentina.............. I

France.................. 3

Germany............... I 3

Italy $\ldots \ldots \ldots \ldots \ldots \ldots \ldots \ldots$ I

Peru.................. 2

United Kingdom. ............ I9

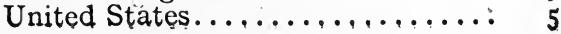

4
I
41
4




\section{IMPORTS AND EXPORTS BY COUNTRIES-Contd.}

(In Millions of Dollars.)

China.

Canada and Newfoundland........ 2

Europe, continent. ............ I 2

French East Indies. . . . . . . . I

Hongkong............... 84

India. .............. $2 \mathrm{I}$

Japan................. 22

Russia.

22 I 8

Straits Settlements. . . . . . . . . 3

United Kingdom. ............. $3_{3}^{6}$

United States.............. I9

DenMARK.

Belgium...............

France.................. 4

Germany .............. 45

Netherlands.............. 3

Norway. .................. ${ }_{2}^{3}$

Rumania................. 2

Russia. . ................ ${ }_{25}$

Sweden................. I5

United Kingdom............ 24

United States............. I 9

72

4

Dutch East Indies.

Australia................ I

France...................

Hongkong. . . . . . . . . . . .

Japan .................

Malacca...................

Netherlands............... 22

Penang.................... 8

Saigon. ................. 4

Singapore. ............... 23

United Kingdom. . . . . . . . . . . 8 


\section{IMPORTS AND EXPORTS BY COUNTRIES-Contd.}

(In Millions of Dollars.)

EgYPT.

Austria-Hungary............ 6

Belgium................. 2

France...................... 7

Germany................. 3

Greece. . ............... I

Italy................... 4

Russia. ................... 3

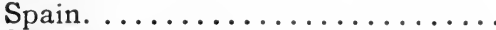

Sweden................ I

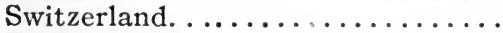

Turkey......................

United Kingdom. ............ 27

Exports.

France.

Algeria.................. 49

Argentina. . . . . . . . . . . . . 4.3

Austria-Hungary ............ I5

Australia.................. $\mathrm{r}_{3}$

Belgium................ 64

Brazil. .................. I6

British South Africa........... 3

Bulgaria............... I

Chile................... I2

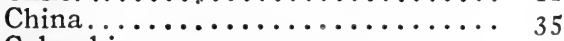

Colombia..............

Cuba...................

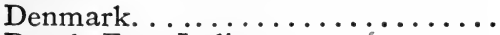

Dutch East Indies. ............ 5

Egypt.................... 9

Germany............. 8 r

Greece.................. 2

Haiti (island). ............. 6

India..................... 40

Indo-China. . ..................

Italy................. $30 \ldots \ldots$

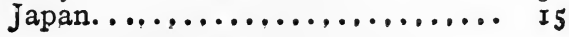

4

$\frac{7}{6}$

3

7
2

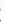

52

8

6

I

I 22

7

2

2

2

3

6

94

2

6

34 


\section{IMPORTS AND EXPORTS BY COUNTRIES-Contd. (In Millions of Dollars.)}

France-Continued.

Mexico.

Imports. Exports.

Netherlands. .............. Io

Norway.

Peru.

IO

Philippine Islands. . . . . . . . . .

Portugal................ I

Rumania................. 7

Russia................... 39

Spain.................. 29

Sweden.................. r 3

Switzerland. ............... 20

Tunis.................... 5

Turkey................... 20

United Kingdom. .............. I

United States.............. 82

Uruguay. ................ 4

Venezuela................ 2

$\mathbf{I}$

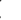

GeRMANY.

Algeria................. 2

Argentina................ 48

Australia................. 29

Austria-Hungary............ I66

Belgium................. 46

Bolivia.

Brazil

British South Africa........... 6

British West Indies............. 2

Bulgaria................. 2

Canada.................... 2

Chile.................... 22

China.................. 7

Colombia................. I

Costa Rica.................. I I

Cuba and Porto Rico........... 3

Denmark................ I8

Dutch East Indies............ 22 


\section{IMPORTS AND EXPORTS BY COUNTRIES - Contd.}

\section{(In Millions of Dollars.)}

Germany-Continued.

Ecuador.

Imports. Exports.

Egypt.................. II

France................. $7^{2}$

French East Indies........... 2

Greece. ................. 3

Guatemala.............. 6

India.................. $5^{\mathrm{I}}$

Italy................... 45

Japan.................. 4

Mexico..................... 3

Netherlands. .............. 46

Norway.

Peru

Portugal.

Rumania

Russia................. I 84

Servia.................. 3

Siam............... I

Spain................. I 8

Sweden................. r 9

Switzerland............... 39

Turkey.

United Kingdom.

United States.............. 2 I 3

Uruguay................ 3

Venezuela................. 2

2

I 4

30

I I

93

14

2

5

9

78

I

I3

28

66

Io

228

107

3

Greece.

Austria-Hungary........... 3

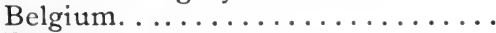

France................. 3

Germany................ ${ }_{2}^{2}$

Italy................ I

Netherlands. . . . . . . . . . . . .

Russia. .................... 6

Turkey................ 2

United Kingdom............. 6

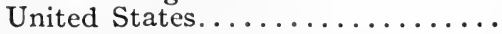

2

$\mathbf{I}$

2

I

2

I

6 
54 STATISTICAL ABSTRACT OF THE WORLD.

\section{IMPORTS AND EXPORTS BY COUNTRIES-Contd.}

\section{(In Millions of Dollars.)}

INDIA.

Imports. Exports.

Aden................... 3

Arabia.................. 224

Australia................. 2

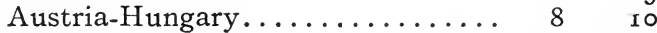

Belgium................ no no

Ceylon. .................. 2 I4

China................... 2 2 I

Egypt..................

France................. 5 , 30

Germany................. 7 7 33

Hongkong................ 5

Italy....................... 3 I I

Japan.................. 3 I

Java.................. 2 I

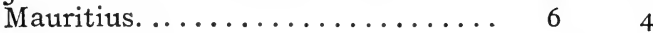

Netherlands............... 2

Persia.................. 2

Russia................. 9 I

South Africa............... 4

Spain...................

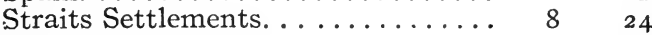

Turkey.................

United Kingdom. .............. I92 I03

United States................ $4 \quad 28$

West Indies................ I

ITALY.

Algeria................ I

Argentina................................ 6

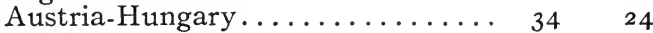

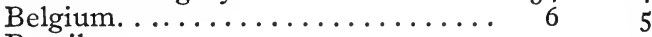

Brazil................... 3

China.................... I

Egypt.................. $2{ }_{2} 6$

France................. $35 \quad 32$

Germany................. $43 \quad 47$ 


\section{IMPORTS AND EXPORTS BY COUNTRIES - Contd.}

(In Millions of Dollars.)

\section{ITALY-Continued.}

Imports. Exports.

India. . . . . . . . . . $\ldots \ldots \ldots$ It 5

Japan................... 2

Netherlands. . . ........... 2

Rumania................ I4

Russia................... 35

Spain. .................. 5

Switzerland............... II

Turkey............... 4

United Kingdom. ........... 55

United States.............. 4 I

23

I

2

52

50

49

JAPAN.

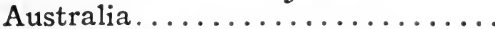

Austria-Hungary............ I

Belgium................. 3

British India.............. 25

Canada and Newfoundland.

China.

20

Dutch East India. ............ ${ }_{2}$

Egypt.

France.

French India. ............. 3

Germany................ I 3

Hongkong............. I

Italy.

I I

Korea.

United Kingdom.

4

United States.

3

I

Mexico.

Belgium................ I

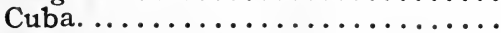

France................ 6

Germany................ 6

Spain. $\ldots \ldots \ldots \ldots \ldots \ldots \ldots \ldots . \ldots \ldots$

United Kingdom. ............ 8

United States.............. 39 


\section{IMPORTS AND EXPORTS BY COUNTRIES-Contd.}

(In Millions of Dollars.)

$$
\text { NATAL. }
$$

Imports. Exports.

Argentina. ............... 3

Australia................. I 5

Cape Colony.............. ${ }_{2}$

Germany................. 3

India.................. ${ }_{2}$

United Kingdom. ........... $4 \mathrm{r}$

United States............. 6

Netherlands.

Austria-Hungary........... 2

Belgium.................. 94

Brazil.................... I2

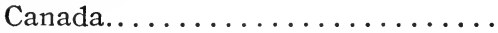

Chile.................... 3

Denmark

Dutch East Indies. ........... I40

France.................. 9

Germany................ 228

Greece................. 4

India................ 22

Italy.................... ${ }_{2}$

Norway. ................ 4

Portugal................ 3

Rumania................ 24

Russia................. 84

Spain. ................. I 5

Sweden................. 8

Turkev................. 2

United Kingdom. ............ 95

United States.............. 94

78

4

4

24

6

360

2

2

4

3

4

4

I

2

4

2

3

3

I84

43 


\section{IMPORTS AND EXPORTS BY COUNTRIES-Contd.}

(In Millions of Dollars.)

New Zealand.

Australia................. Io

Imports. Exports.

Germany............. I

India. ............. I

South Africa.

United Kingdom. ............ 33

United States............. 6

Norway.

Belgium............... $3 \quad 2$

Denmark................ 6

France................ I

Germany................ $21 \quad 7$

Netherlands............... $4 \quad 3$

Russia................. $88_{2}$

Spain. ............. I 3

Sweden................. 74

United Kingdom. ........... 2 I 19

United States.............. 3

Philippine Islands.

British East Indies. .......... 3

China...................

France.............. I

French Indo-China. .......... 8

Germany................ 2

Japan. ............

Spain. .................. 2

United Kingdom. ............ 5

United States.............. 4

2

3

59

4 I 3 


\section{IMPORTS AND EXPORTS BY COUNTRIES-Contd.} (In Millions of Dollars.)

Portugal.

Belgium. ................

Brazil. .................... 3

France.................. 6

Germany................ Iо

Italy................ I

Spain................... 7

Sweden and Norway........... I

United Kingdom............. 19

United States.............. 4

Rumania.

Austria-Hungary..............

Belgium.

France.

Germany . . . . . . . . . . . .

\section{Netheria}

Russia.

I 5

Switzerland.

Turkey. ..................

United Kingdom.

Russia.

Austria-Hungary............ I 3

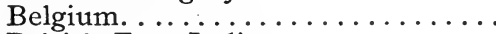

5 I I

British East Indies. ............

China.

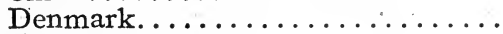

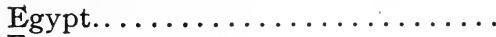

Germany................ Iо

Netheriands....................... 5

Netherlands. ............... 4

Norway. 


\section{IMPORTS AND EXPORTS BY COUNTRIES-Contd.} (In Millions of Dollars.)

\section{Russia-Continued.}

Persia

Imports. Exports.

Rumania................. I

Spain................ I

I 2

Sweden..................

Switzerland. .............. 3

Turkey................. 4

United Kingdom. ......... 53

United States............. I8

Servia.

Austria-Hungary............. Germany.

Siam.

Hongkong............... 4

Singapore.

United Kingdom.

I I

$8 \mathrm{I}$

2

IO 8

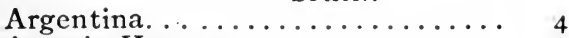

SPAIN

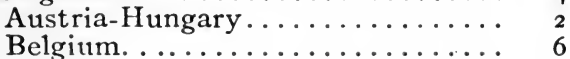

2

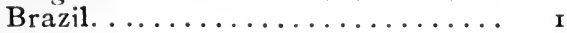

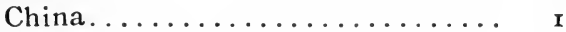

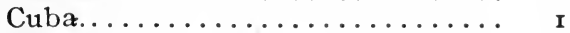

Egypt..................... 3

France................. 30

Germany............... ${ }^{17}$

Italy................ 4

Mexico..................

Netherlands............. 2

Norway.................... 3

Philippine Islands. . . . . . . . 3

Portugal. ............... 8

Russia.................. 6

Io

Sweden................. 2

Switzerland. .............. 4

United Kingdom............. $3^{6}$

United States.............. 22 


\section{IMPORTS AND EXPORTS BY COUNTRIES-Contd.} (In Millions of Dollars.)

Straits Settlements.

Imports. Exports.

China................. 2

22

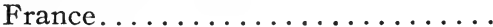

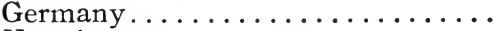

Hongkong. ...............

India. ................... 3I

Italy. 2

Japan.

$3 \quad 4$

I 5

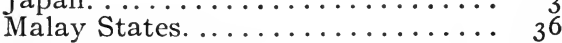

Philippine Islands. . . . . . . . . .

Siam.

United Kingdom. ............

United States..............

SWEDEN.

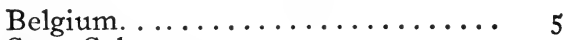

Cape Colony. ...............

Denmark...................... I7

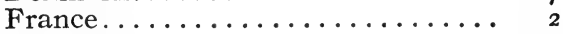

Germany................. 53

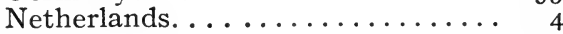

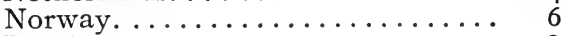

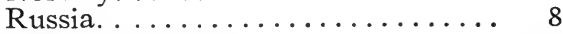

United Kingdom............ 34

United States.............. 3

Switzerland.

Argentina. ..............

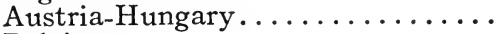

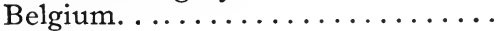

Brazil.

Bulgaria, Rumania, and Servia.... 3

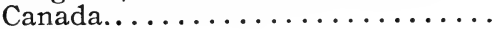

China................... 3

Egypt.................. 3

France................. $4^{2}$ 
STATISTICAL ABSTRACT OF THE WORLD. 6I

\section{IMPORTS AND EXPORTS BY COUNTRIES-Contd.}

(In Millions of Dollars.)

SWITZERLAND-Continued.

Imports. Exports.

Germany.................. $6_{3}$

India.

$\begin{array}{rr}3 & \\ 1 & 2\end{array}$

Italy.

34

Japan

2

Netherlands.

Russia.................... I 12

Spain.

3

United Kingdom. ............. Io 10

United States.

\section{2} I I 5

\section{Tunis.}

France................... 8

Italy............... I

United Kingdom. ........... I

4

I

$\mathbf{I}$

TURKEY.

Austria-Hungary............ 2 I

Belgium................. 3

Bulgaria................. 5

France.................. r 3

Germany................ 2

Greece.................. 2

Italy................... 6

Netherlands. ............ I

Persia................... 3

Rumania................. 3

Russia.................. 9

United Kingdom............ 37 2 I 


\section{IMPORTS AND EXPORTS BY COUNTRIES-Contd.}

\section{(In Millions of Dollars.)}

\section{United Kingdom.}

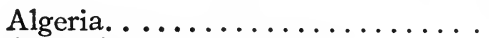

Imports. Exports.

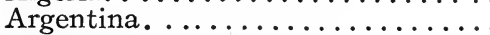

3

2

Australia

68

29

Austria-Hungary............

Belgium................. I 29

Brazil. .................... 30

British South Africa. . .......... 27

$96 \quad 95$

British West Indies. ........... r. ro

Bulgaria................. 2

Canada and Newfoundland......... Ir 5

Chile.................... 22

China.................. I

Colombia............... ${ }_{2}$

Denmark. ................. ${ }_{7} 6$

Dutch East Indies. . . ......... I

Egypt....................... $6_{7}$

France................... 246

Germany................ I64

Greece. ................. 8

India. . ................. r 40

Italy.................. I 7

Japan. .................. 9

Mexico................. I

Netherlands. . . . ........... I70

New Zealand............... 53

Norway and Sweden.......... 73

Peru.

Philippine Islands

Portugal.

II

Rumania

I 7

Russia.

37

Spain

I 25

Straits Settlements........... 29

Turkey.

United States. . . . . . . . . . . 6

Uruguay.

9




\section{IMPORTS AND EXPORTS BY COUNTRIES-Contd.}

(In Millions of Dollars.)

United States.

Argentina. . . . . . . . .

Australia and New Zealand....... 7

Austria-Hungary............. II

Belgium................. 23

Brazil. .................. 67

British Africa...............

British East Indies. . . . . . . 52

British Guiana. ............. 3

British West Indies............ I 3

Canada and Newfoundland. . . .... $5^{6}$

Chile................... 9

China................... 27

Colombia................ 4

Costa Rica................. 4

Cuba...................... 63

Denmark.

Dutch East Indies. . .......... I6

Ecuador................. 2

Egypt................. II

France..................... 90

Germany.................. I 20

Greece. ................ I

Guatemala................ 2

Haiti................... I

Italy. .................. ${ }_{3}^{6}$

Japan.................. 44

Mexico.................. 4r

Netherlands. . . . . . . . ... 23

Nicaragua. .............. ${ }_{2}^{2}$

Peru................... 3

Philippine Islands. . . . . . . $\ldots \ldots \ldots$ I

Portugal. ................ 4

Russia.

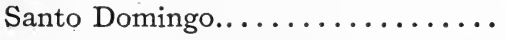

Exports.

I I

33

7

47

I I

34

5

IO

I I 7

4

I 9

4

2

20

I 6

I

I

75

I 9 I

3 


\title{
IMPORTS AND EXPORTS BY COUNTRIES-Contd.
}

\author{
(In Millions of Dollars.) \\ United States-Continued.
}

Spain. .................. $8{ }^{\text {Imports. }}$ I 8

Sweden and Norway........... 5 ro

Switzerland............... 2 I

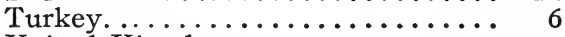

United Kingdom. .............. r

Uruguay................. 3

Venezuela. ................ 5

$5 \times 7$

$3 \quad 1$

52

URUGUAY.

Argentina................. 3 5

Belgium................. I

Brazil................... 2

France................... 2 5

Germany................... 3

Italy.................. 2

Spain.................. 2

United Kingdom............. 6

United States............... a 
STATISTICAL ABSTRACT OF THE WORLD. 65

\title{
IMPORTS AND EXPORTS BY COMMODITIES.
}

\author{
(In Millions of Dollars.)
}

Argentina.

Imports.

Food............. I 2

Drink.......... 7

Textiles and clothing . 39

Oil............. 4

Chemicals......... 5

Timber and manufactures.......... 9

Iron and manufactures 27

Other metals and manufactures........ 4 Glass and pottery..... I 2 Tobacco.......... 4
Animals and products. 109 Agricultural products. 103 Forest products..... 3

\section{Australia.}

Wheat and flour...... r.3 Sugar........... 5 Tea............. 3 Spirits.......... 4 Clothing. ......... I 8 Cotton and linen goods ro Woolen goods........ 7 Silk goods. ......... 3 Machinery.......... ro Agricult. implements. . 2 Metal manufactures. .. 9 Iron and steel. ....... 7 Timber........... 5 Arms and explosives. . 4 Paper............ 4 Chemicals. . . ...... 4 Railway materials. ... 2
Exports 


\section{IMPORTS AND EXPORTS: COMMODITIES-Contd.}

(In Millions of Dollars.)

Austria-Hungary.

Imports.

Cotton. ......... 37

Coal and coke....... 20

Maize (corn)........ 6

Tobacco........... I I

Coffee............ 8

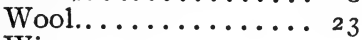

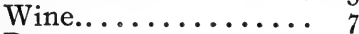

Rye............ 3

Eggs........... 7

Silk and manufactures. I 6

Copper........... 6

Flax and jute....... I I

Machinery......... Io

Hides............ Io
Exports.

Sugar........... 33

Eggs. ............ 2 I

Wood and manufact... ro

Barley........... I 8

Lignite. .......... I3

Malt............ I r

Leather and manufact. 9

Cattle.......... 20

Horses. . . . . . . . I I

Glass and manufact... I I

Wool manufactures... Io

Belgium.

Wheat.......... 53

Corn............ I 2

Barley........... I I

Timber.......... 28

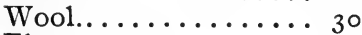

Flax................. 27

Cotton........... I 5

Hemp........... 4

Chemicals......... I4

Drugs........... 8

Coal. ........... I 3

Diamonds......... I 6

Iron and ore........ Io

Other metals......... I I

Minerals.......... I 8

Machinery......... Io

Rubber........... I I

Oils.
Coal and coke...... 24

Linen yarn......... I 7

Flax............ ${ }_{2} \mathrm{O}$

Wool and yarn......... ${ }_{2}^{6}$

Wheat........... Io

Sugar............. 6

Hides............ $\mathrm{I}^{2}$

Glass................ $\mathrm{I}^{8}$

Machinery............... ${ }_{2}$ o

Iron and steel. ...... $3^{2}$

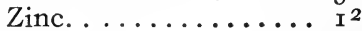

Diamonds. ......... I 7

Rubber........... 9 


\section{IMPORTS AND EXPORTS: COMMODITIES-Contd.}

\section{(In Millions of Dollars.)}

\section{BrazIL.}

Coffee. .................... 96

Rubber.................... 49

Cotton. .................. 7

Hides...................... 9

Cocoa.................... 5

\section{Chile.}

Minerals.......... 7

Textiles.......... I 3

Oils............. 7

Paper.

Machinery and tools... 5
Exports.

Nitrates......... 42

Copper........... 6

Iodine............ I

Wheat.......... I

Silver........... I

\section{China.}

Opium. ......... 30 Tea. .......... r 8

Cotton goods......... 86

Woolen goods. ....... 3

Metals........... ro

Coal. ............ 6

Silk and manufactures 50

Hides and skins. . . ... 6

Paper............ 2

Oil.............. Iо

Rice............ 6

Sugar............ 10

Fish............ 3

Cotton........... 9

Cuba.

Metals and manufact.. 4 Sugar......... 42

Chemicals......... 3

Textiles and manufact. Io

Fruit............ 3

Animals and products. 9

Tobacco............. 26

Food............. 22 


\section{IMPORTS AND EXPORTS: COMMODITIES-Contd.}

(In Millions of Dollars.)

\section{DenMark.}

Textiles. ................ I9

Metals and hardware........... I4

Wood and manufactures......... 8

Coal. ................... ro

Animals.............. I

Provisions................ 23

Cereals.................. 20

I

8

8

78

\section{Egypt.}

Animal products............ 9

Cereals and vegetables. ........ ro

Spirits, oils, etc............ 5

Wood and coal............... I 3

Textiles................. $3^{\mathrm{I}}$

Metals and manufactures......... I 5

Tobacco................. 3

I3

2

84

2

\section{France.}

Imports.

Nine. ............. 36 Wool, raw......... 76 Cereals........... 35 Silk, raw. . ....... 64 Cotton, raw. ....... 73 Timber........... 33 Hides and furs. ..... 34 Oil seeds. . . . . . . . 44 Coffee............2 23 Coal and coke....... 50
Fxports. Wool manufactures... 44 Silk manufactures. . . 49 Cotton manufactures . 36 Wine............... 45 Silk, raw. . ....... 29 Wool, raw......... 45 Leather. . ........ 23 Linen........... 20 Skins and furs....... 24 Chemicals......... I9 


\section{IMPORTS AND EXPORTS: COMMODITIES-Contd.}

\section{(In Millions of Dollars.)}

Germany.

\begin{tabular}{|c|c|c|}
\hline & & \\
\hline Animal products. ...... & 53 & \\
\hline Food. . . . . . . . . & 422 & 122 \\
\hline Fuel. .. & $4 \mathrm{I}$ & 72 \\
\hline Oil. ............. & $9^{2}$ & I 2 \\
\hline Chemicals and drugs $\ldots \ldots \ldots \ldots \ldots$ & 88 & I I 2 \\
\hline Stone, clay, and glass products. .... & 17 & 50 \\
\hline Metals and manufactures......... & I 73 & $23 I$ \\
\hline Wood and manufactures........... & 79 & $3^{8}$ \\
\hline Paper................. & 9 & 33 \\
\hline Leather and manufactures........ & IOI & 84 \\
\hline Textiles. $\ldots \ldots \ldots \ldots \ldots \ldots \ldots \ldots$ & $35 \mathrm{I}$ & 306 \\
\hline Machinery and tools. . . . . . . . . & 22 & 89 \\
\hline Hardware. . . . . . . . & 7 & 45 \\
\hline
\end{tabular}

Greece.

Imports.

Agricultural products . 8

Yarn and cloth...... 4

Minerals.......... 3

Timber.......... 2

Metals........... I

Chemicals......... I

Animals and products. 2

Fish............. I
Imports. Exports.

53

422

72

I 2

50

$3 \mathbf{I}$

38

33

84

306

89

45

\section{INDIA.}

Cotton goods........ I $\mid$ Rice. ........... 6

Metal goods......... 3 r 3 Wheat............ r

Sugar...................... 8

Oil. ............. I

Machinery......... I

Agricultural products . 8

Minerals.......... 4

Wines............ 2

Olive-oil.......... I

Animal products..... I

Timber......... I

Opium........... 3

Oil seeds. ........ 5

Jute.......... 7

Tea............. 2 


\section{IMPORTS AND EXPORTS: COMMODITIES-Contd.} (In Millions of Dollars.)

ITALY.

Imports.

Wheat.................... 38

Cotton....................... 37

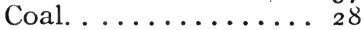

Timber........... I 2

Wool............. I I

Silk, raw............ 2 I

Machinery........ I 4

Fish........... 9

Iron and steel. ...... 4

Coffee. ............

Hides. . . .........

Tobacco. ...........

Horses. . . . . . . . . . .

Petroleum......... 3

Olive-oil.

Rice............

Wheat and flour......

Sugar..............

Cotton.

Cotton manufactures. .

Wool and manufact. ..

Petroleum...........

Iron manufactures....
Exports.

Silk, raw. . . . . . . 93

Wine........... I 3

Olive-oil. ..........

Eggs............ 8

Hemp and flax..... 8

Sulphur.......... 9

Marble.......... 5

Meat........... 3

Skins.......... 5

Zinc ore.......... 2

JAPAN.

26 Cotton manufactures . I9

8 Silk, raw........ 4 I

I I Silk manufactures .... I6

35 Coal....................... Io

5 Matches......... 4

7 Copper........... 8

6 Tea............ 7

ro Rice............. 3

\section{Mexico.}

Minerals.......... I I

Vegetables......... 7

Machinery..........6 6

Textiles.......... 5

Animal substances. ... 3

Liquors.......... 2

Chemicals and drugs .. 2
Gold............. 6

Silver........... 40

Copper........... I 2

Lead............. 3

Coffee........... 5

Henequen. . ........ I6

Lumber. . . . . . . . 2

Vegetable products ... 8

Animals and their products.......... 


\section{IMPORTS AND EXPORTS: COMMODITIES-Contd.}

\section{(In Millions of Dollars.)}

NetherLANDS.

Iron and steel.

Imports. Exports.

Textiles.

Grain and flour.

I 3

2 I

\section{NoRWAY.}

Animals and their products........

Breadstuffs.

Groceries .

Fruits.............. I

Spirits................ 2

Yarn, rope, etc. ............ 5

Manufactured textiles.......... 7

Hair and skins. ............ 2

Tallow, oil, tar, etc. ......... 6

Timber................... 3

Paper. ............. I

Minerals................ ro

Metals.................. 3

Manufactured metals. ......... 6

Vessels, carriages, and machinery . . 7

\section{PERU.}

Imports.

Cotton goods......... 2

Woolen goods. ....... I

Metal manufactures... 5

Machinery......... I

Spirits.

Exports.

\section{Portugal.}

Coal. ............. 4

Cotton............ 4

Iron and its manufact. 4 Codfish............ 4 Wheat............. 3

Sugar............. 2

Wool. ..............

Hides and skins. ....

Machinery......... 2

Wine........... ro

Cork. ............... 3

Fish. .............

Cotton goods......... 2

Copper ore......... I 


\section{IMPORTS AND EXPORTS: COMMODITIES-Contd.}

\section{(In Millions of Dollars.)}

Philippine Islands.

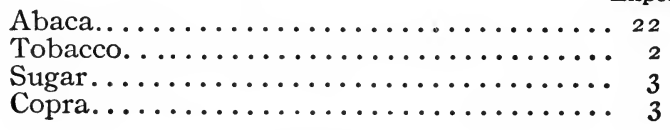

Rumania.

Cereals................. r

Textiles................ 22

Metals and their manufactures..... I I

Hides and leather........... 2

Animals and their products....... 2

Chemicals and drugs.......... 3

Exports.

\section{Russia.}

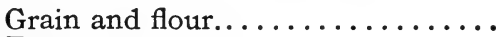

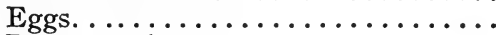

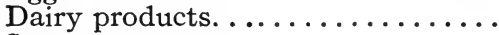

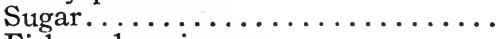

Fish and caviar. ................

Timber and its manufactures......

Petroleum..................

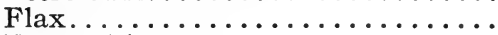

Fur and leather. ............ ro

Tea.................... r 3

Wine, ale, and spirits.......... 7

Raw cotton............... 49

Metals................... I4

Coal and coke.............. II

Wool................... I

Silk.................. 9

Machinery.................. $3^{\circ}$

Metal goods............... I4

Cotton goods............... I 2 2 3 


\section{IMPORTS AND EXPORTS: COMMODITIES-Contd.}

\section{(In Millions of Dollars.)}

\section{Servia.}

Imports. Exports

Agricultural products.

Animals and their products........

Hides, skins, and leather........ I

Wool and its manufactures. ....... I I

Metals................. I

Clothing............. I

\section{Spain.}

Stone, minerals, glassware, and

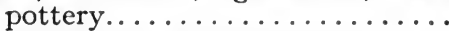

Metals and their manufactures. ....

Drugs and chemicals.......... 20

Cotton and its manufactures....... 22

Other vegetable fibres and manufact. 5

Wool and its manufactures....... 5

Silk and its manufactures......... 5

Paper and its manufactures....... 3

Timber and its manufactures...... I 2

Animals and their products....... I6

Machinery, vehicles, and vessels.... I 14

Food, sugar, and wine......... 24

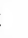




\section{IMPORTS AND EXPORTS: COMMODITIES-Contd.}

(In Millions of Dollars.)

Switzerland.

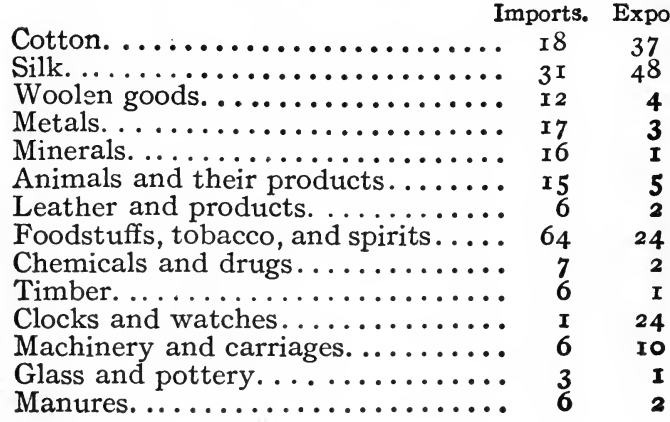

Transvaal.

Imports.

Manufactured metals. . I 3 Clothing........... I I Provisions.......... 8

Grain and flour...... 4 Wood and manufact... 4 Leather manufactures. 3 Chemicals......... 2 Animals........... 2 Coal. ............. I

TURKEY.

Sugar............. Sheeting, quilts, and prints. .......... 3 Wheat and flour...... 9 Rice............ 4 Cotton yarn..........

9 Grapes........... 8 Silk............ 7 Mohair........... 4 Silk cocoons........ 4 Opium.......... 3 4 Skins and hides..... 3 
STATISTICAL ABSTRACT OF THE WORLD. 75

\section{IMPORTS AND EXPORTS: COMMODITIES-Contd.}

\section{(In Millions of Dollars.)}

TURKEY-Continued.

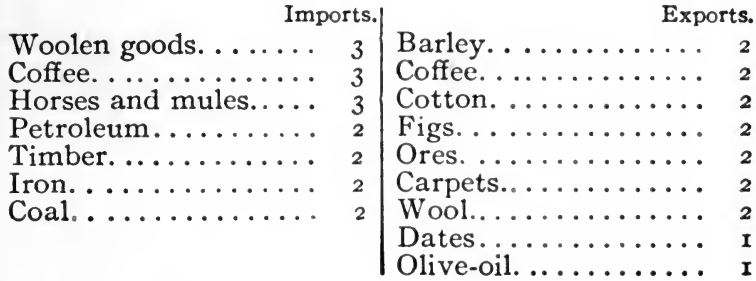

\section{United Kingdom.}

Grain and flour.......348 Cotton, raw.......274 Wool, raw..........102 Meat............. 86 Sugar............ 92 Butter and cheese. . . I 47 Lumber. ......... I 8 Silk manufactures. . . 64 Flax, hemp, and jute. . 57 Tea............. 47 Wool manufactures... 57 Live animals. ....... 52 Oils. .............. 54 Chemicals, dyes, etc. .. 47 Oil seeds. ........... 37 Fruit and hops...... 59 Leather and hides.... 40 Wine............. I9 Metals and ores...... I I Coffee. ........... I8 Tobacco........... 22
Cotton manufactures. .420 Wool manufactures. . . I 20 Linen and jute manuf.. 45 Clothing........... 36 Iron and steel manuf: . I65 Copper manufactures.. I6 Machinery.......... 106 Coal. ............ I35 Chemicals, dyes, etc.. 69 


\section{IMPORTS AND EXPORTS: COMMODITIES-Contd.}

\section{(In Millions of Dollars.)}

\section{United States.}

Imports.

Sugar............ $7^{2}$

Hides............. 52

Chemicals and drugs. . 65

Coffee............6.63

Raw silk........... 46

Cotton manufactures. . 50 Rubber........... 42

Fibres, manufactures. . 40 Silk manufactures. ... $3^{2}$ Fibres, raw. ........ 38 Wood and manufact... 27 Iron and steel and manufactures........ 27 Wool, raw.......... 25 Fruit............. 24 Tin............. 2 I Tea............. 18 Wool manufactures... I 8 Tobacco............ 20 Liquors........... 17 Diamonds. ......... 34 Copper............ I8
Exports.

Breadstuffs. ........ I49 Raw cotton. ........37 I Provisions........... I 76 Iron and steel and manufactures. ..... I I 2 Petroleum........ 79 Copper and manufact. . $5^{8}$ Wood manufactures... 65 Animals.......... 48 Tobacco........... 35 Leather and manufact. 34 Cotton manufactures. . 22 Coal. ........... 28 Agricul. implements... 23 Fruit........... 2 I Chemicals and drugs. . I4

\section{URUGUAY.}

Food and drink...... Textiles. Clothing. Raw material and machinery.......
Animal products..... 35 Agricultural products . 


\section{INDEX.}

PAGE

Alcohol, consumption of. ............. $4^{2}$

Algeria, imports and exports by countries. . . . . 45

Aluminum, production of. ............. 22

Antimony, production of. .............. 22

Areas:

Cultivated................... 43

Wooded........................ $4^{2}$

Areas of continents.................. I

Areas of countries. ................. 2

Argentina:

Imports and exports by commodities....... 65

Imports and exports by countries. . . . . . . . 46 Arsenic, production of. ................ 22 Asphaltum, production of. ............. 23 Attendance a. school, proportion............ I 3 Australia:

Imp rts and exports by commodities. . . . . . 65

Imports and exports by countries. . . . . . . 46 Austria-Hungary:

Imports and exports by commodities. . . . . . 66

Imports and exports by countries. . . . . . . 46 Barley, production of. ................. 30 Beer, consumption of. . . . . . . . . . . . $4^{2}$ Belgium:

Imports and exports by commodities. . . . . . 66

Imports and exports by countries. . . . . . 47

Birth-rates. . .................... I 5

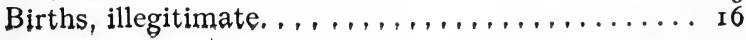


PAGE

Blind. ...................... I7

Borax, production of............... 24

Brazil, exports by commodities...........667

Breadwinners ..................... I 7

Bulgaria, imports and exports by countries. ..... 48

Canada, imports and exports by countries. . . . . . 48

Cape Colony, imports and exports by countries. .. . 49

Cattle......................... 40

Ceylon, imports and exports by countries. . . . . . 49

Chile:

Imports and exports by commodities. . . . . . . 67

Imports and exports by countries. . . . . . . . 49 China:

Imports and exports by commodities. . . . . . . 67

Imports and exports by countries. . . . . . . 50

Cities, population of. ............... 4

Coal, production of. ................ 24

Cocoa:

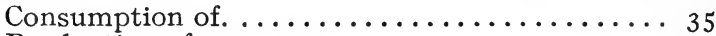

Production of. ..................... 35

Coffee:

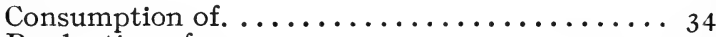

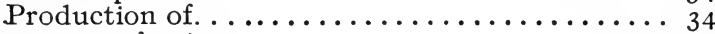

Commerce, foreign................... 44

Conjugal condition................. I4

Continents:

Areas of...................... I

Population of $\ldots \ldots \ldots \ldots \ldots \ldots \ldots \ldots$ I

Copper:

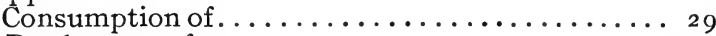

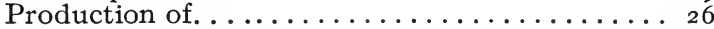

Corn, production of. ................ 30

Cotton:

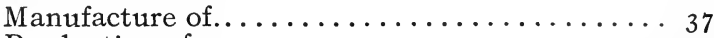

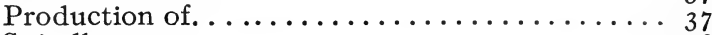

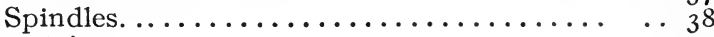

Countries:

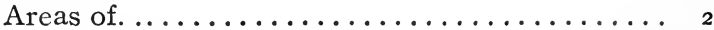

Population of................... 2

Cuba, imports and exports by commodities: : : : : : 67 
PAGE

Cultivated area..................... 43

Deaf-mutes. ...................... I 7

Death-rates....................... I5

Debt.......................... I 9

Denmark:

Imports and exports by commodities. . . . . . . 68

Imports and exports by countries. . . . . . . . $5^{\circ}$

Dutch East Indies, imports and exports by coun-

tries........................ $5^{\circ}$

Egypt:

Imports and exports by commodities. . . . . . 68

Imports and exports by countries. . . . . . . $5^{\text {I }}$

Expenditures...................... 19

Exports........................ 44

Exports by commodities. . . . . . . . . . . 65-76

Argentina, 65; Australia, 65; Austria-Hungary, 66; Belgium, 66; Brazil, 67; Chile, 67; China, 67; Cuba, 67; Denmark, 68; Egypt, 68; France, 68; Germany, 69; Greece, 69; India, 69; Italy, 70; Japan, 70; Mezico, 70; Netherlands, 71; Norway, 7I; Peru, 7I; Portugal, 7I; Philippine Islands, 72; Rumania, 72; Russia, 72; Servia, 73; Spain, 73; Sweden, 73; Switzerland, 74; Transvaal, 74; Turkey, 74; United Kingdom, 75; United States, 76; Uruguay, 76.

Exports by countries................ 45-64 Algeria, 45; Argentina, 46: Australia, 46; Austria-Hungary, 46; Belgium, 47; Bulgaria, 48; Canada, 48; Cape Colony, 49; Ceylon, 49; Chile, 49; China, 50; Denmark, 50; Dutch East Indies, 50; Egypt, 5r; France, 5I; Germany, 52; Greece, 53; India, 54; Italy, 54; Japan, 55; Mexico, 55; Natal, 56; Netherlands, 56; New Zealand, 57; Norway, 57; Philippine Islands, 57; Portugal, 58; Rumania, 58; Russia, 58; Servia, 59; Siam, 59; Spain, 50; Straits Settlements, 60; Sweden, 60; Switzerland, 60; Tunis, 6r; Turkey, 6r; United Kingdom, 62; United States, 63 ; Úruguay, 64 .

Fisheries. 
Flax, production of................ 35

Foreign-born:

Proportion of. ................. I 2

Proportion of each leading nationality....... I3 Foreign commerce. .................. 44 France:

Imports and exports by commodities......... 68

Imports and exports by countries.......... 5 I Germany:

Imports and exports by commodities........69

Imports and exports by countries............ $5^{2}$

Goats. ......................... 40

Gold, production of. .................. 28

Graphite, production of. . . . . . . . . . 22

Greece:

Imports and exports by commodities. . . . . . . 69

Imports and exports by countries......... 53

Hogs. . . ...................... 40

Homicide, convictions for. ............ I 8

Horses......................... 40

Illegitimate births................. I6

Imports. . . . . . . . . . . . . . . . 44

Imports by commodities. . . . . . . . . . 65-76

Argentina, 65; Australia, 65; Austria-Hungary, 66; Belgium, 66; Brazil, 67; Chile, 67; China, 67; Cuba, 67; Denmark, 68; Egypt, 68; France, 68; Germany, 69; Greece, 69; India, 69; Italy, 70; Japan, 70; Mexico, 70; Netherlands, 7I; Norway, 7 r ; Peru, 7 I ; Portugal, 7 I ; Philippine Islands, 72; Rumania, 72; Russia, 72; Servia, 73; Spain, 73; Sweden, 73; Switzerland, 74; Transvaal, 74; Turkey, 74; United Kingdom, 75; United States, 76; Uruguay, 76.

Imports by countries. . . . . . . . . . . . Algeria, 45; Argentina, 46; Australia, 46; Austria-Hungary, 46; Belgium, 47; Bulgaria, 48; Canada, 48; Cape Colony, 49; Ceylon, 49; Chile, 49; China, 50; Denmark, 50; Dutch East Indies, 50; Egypt, 5I; France, 5I; Germany, 52 ; Greece, 53; India, 54; Italy, 54; Japan, 55; 
PAGE

Mexico, 55; Natal, 56; Netherlands, 56; New Zealand, 57; Norway, 57; Philippine Islands, 57; Portugal, 58; Rumania, 58; Russia, 58; Servia, 59; Siam, 59; Spain, 59; Straits Settlements, 60; Sweden, 60; Switzerland, 60; Tunis, 61; Turkey, 61; United Kingdom, 62; United States, 63; Uruguay, 64 .

Increase of population, rate of. . . . . . . . I I India:

Imports and exports by commodities........66 69

Imports and exports by countries. . . . . . . 54

Insane and idiots. ................. I 7

Interest. . .................. I9

Iron ore, production of. . . . . . . . . . . 26

Italy:

Imports and exports by commodities. . . . . . 70

Imports and exports by countries......... 54 Japan:

Imports and exports by commodities......... 70

Imports and exports by countries.......... 55

Languages, proportion of the population speaking

certain................... I

Literate, proportion of. ............. I 3

Manganese ore, production of. ........... 23

Marriage-rates. .................. 15

Married, proportion of. .............. I4

Mexico:

Imports and exports by commodities........ 70

Imports and exports by countries.......... 55

Natal, imports and exports by countries....... 56

Netherlands:

Imports and exports by commodities....... $7 \mathrm{I}$

Imports and exports by countries.......... 56

New Zealand, im orts and exports by countries. . 57

Norway:

Imports and exports by commodities....... $7 \mathrm{I}$

Imports and exports by countries......... 57

Oats, production of. . .................. 30

Peru, imports and exports by commodities, . . . , , 7 
Petroleum, production of. .............. 24

Philippine Islands:

Imports and exports by commodities........ 72

Imports and exports by countries........ 57

Pig iron, production of. $\ldots \ldots \ldots \ldots \ldots \ldots \ldots \ldots$

Population:

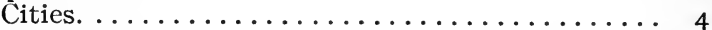

Continents................. I

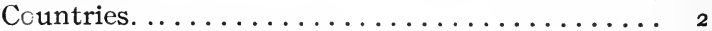

Population, rate of increase............ I I Portugal:

Imports and exports by commodities........ 7 I

Imports and exports by countries.......... $5^{8}$

Potatoes, production of. .............. 29

Quicksilver, production of. ........................

Race, proportion of the population of each. ...... I

Railway mileage..................... 20

Religious adherents.................. I6

Revenue....................... I9

Rumania:

Imports and exports by commodities....... 72

Imports and exports by countries......... $5^{8}$

Russia:

Imports and exports by commodities........ 72

Imports and exports by countries.......... $5^{8}$

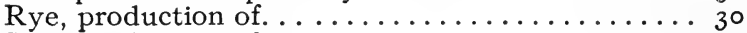

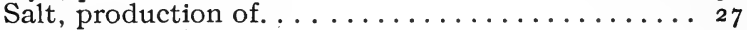

School attendance.................... I 3

Servia:

Imports and exports by commodities........ 73

Imports and exports by countries......... 59

Sex, proportion of each............... I I

Sheep........................ 40

Shipping, tonnage of. ............... 2 I

Siam, imports and exports by countries. . . . . . . 59 Silk:

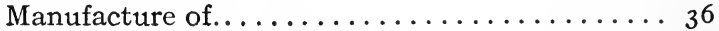

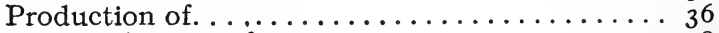

Silver, production of. ............... 28

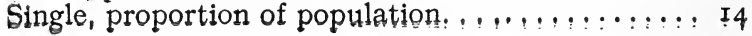


PAGE

Spain:

Imports and exports by commodities....... 73

Imports and exports by countries......... 59

Steel, production of. ................. 27

Straits Settlements, imports and exports by coun-

tries.......................... 60

Sugar:

Consumption of.

Importations to United States. ............ 33

Sugar-bret, production of...................... $3^{2}$

Sugar-cane, production of. . . . . . . . . . $3^{2}$

Sulphur, production of............... 24

Sweden:

Imports an 1 exports by commodities....... 73

Imports and exports by countries..........60 Switzerland:

Imports and exports by commodities........ 74 Tea:

Imports and exports by countries.........6. 60

Consumption of. . . . . . . . . . . . . 35

Production of. ...................... 34

Tin, production of . . . . . . . . . . . . 23

Tobacco, production of. ................ $3^{\mathrm{r}}$

Transvaal, imports and exports by commodities. . 74

Tunis, imports and exports by countries........ 6 I Turkey:

Imports and exports by commodities........ 74

Imports and exports by countries.......... 6 I United Kingdom:

Imports and exports by commodities........ 75

Imports and exports by countries.........62 62 United States:

Imports and exports by commodities........76

Imports and $\exp \mathrm{rts}$ by countries.......... $6_{3}$ Uruguay:

Imports and exports by commodities....... 76

Imports and exports by countries.........64

Wheat, production of. .................. 30

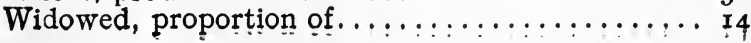


Wine:

PAGE

Consumption of. ............... 4 I Production of. ........................ 42 Wooded area................... 42 Wool, production of. . . . . . . . . . . . . . . 39 Zinc, production of. $\ldots \ldots \ldots \ldots \ldots \ldots \ldots \ldots \ldots \ldots$

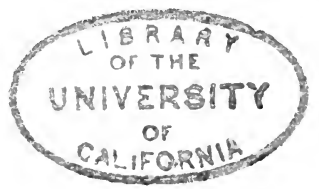






$$
\frac{\pi}{t} c
$$


THIS BOOK IS DUE ON THE LAST DATE STAMPED BELOW

AN INITIAL FINE OF 25 CENTS WILL BE ASSESSED FOR FAILURE TO RETURN THIS BOOK ON THE DATE DUE. THE PENALTY WILL INCREASE TO 50 CENTS ON THE FOURTH DAY AND TO \$1.00 ON THE SEVENTH DAY OVERDUE.

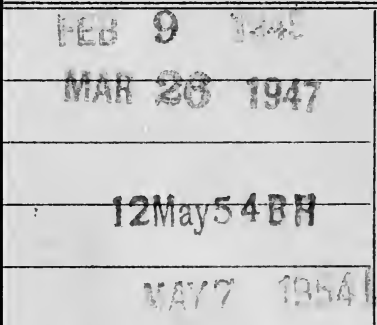




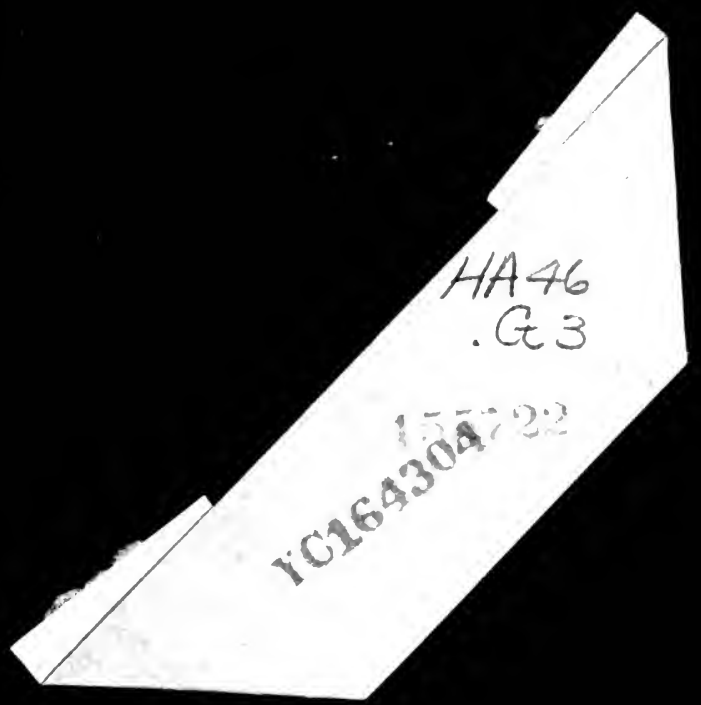


